\title{
Nanomaterial-Based Biosensors using Field-Effect Transistors: A Review
}

\author{
T. Manimekala $^{1,2} \cdot$ R. Sivasubramanian ${ }^{2} \cdot$ Gnanaprakash Dharmalingam $^{1}$ (i)
}

Received: 29 September 2021 / Accepted: 1 February 2022 / Published online: 26 February 2022

(c) The Minerals, Metals \& Materials Society 2022

\begin{abstract}
Field-effect transistor biosensors (Bio-FET) have attracted great interest in recent years owing to their distinctive properties like high sensitivity, good selectivity, and easy integration into portable and wearable electronic devices. Bio-FET performance mainly relies on the constituent components such as the bio-recognition layer and the transducer, which ensures device stability, sensitivity, and lifetime. Nanomaterial-based Bio-FETs are excellent candidates for biosensing applications. This review discusses the basic concepts, function, and working principles of Bio-FETs, and focuses on the progress of recent research in Bio-FETs in the sensing of neurotransmitters, glucose, nucleic acids, proteins, viruses, and cancer biomarkers using nanomaterials. Finally, challenges in the development of Bio-FETs, as well as an outlook on the prospects of nano Bio-FET-based sensing in various fields, are discussed.
\end{abstract}

Keywords Bio-FET $\cdot$ nanomaterial $\cdot$ silicon nanowire $\cdot$ carbon nanotubes $\cdot$ metal oxide $\cdot$ dopamine $\cdot$ glucose $\cdot$ nucleic acid

\section{Introduction}

Biosensors have received great attention in fields including clinical diagnostics, environmental monitoring, sports surveillance, agriculture, and the marine sector. ${ }^{1-3}$ Biosensors are devices that convert a biological response into quantifiable electrical signals. ${ }^{4}$ Several biosensors are available in the market that are useful in health monitoring, such as glucose sensors and oxygen sensors (Clark electrode). ${ }^{5,6} \mathrm{In}$ addition, enzyme-based sensors, ${ }^{7}$ immunosensors, ${ }^{8}$ and $\mathrm{pH}$ biosensors ${ }^{9}$ have been increasingly reported in recent years. An example is the rapid antigen testing kit for the detection of the SARS-CoV-2 virus. ${ }^{10}$ Multiple sensing techniques are available for the detection of biomolecules, such as surface plasmon resonance (SPR), micro-cantilever, electrochemical, colorimetric, fluorescence and chemiresistive-based sensors,,${ }^{11-13}$ with their pros and cons. ${ }^{14}$ For long-term health

Gnanaprakash Dharmalingam

dgp@psgias.ac.in

1 Plasmonic Nanomaterials Laboratory, PSG Institute of Advanced Studies, Peelamedu, Coimbatore, Tamilnadu 641004, India

2 Electrochemical Sensors and Energy Materials Laboratory, PSG Institute of Advanced Studies, Peelamedu, Coimbatore, Tamilnadu 641004, India care monitoring, it is evident that a device with high sensitivity, selectivity, and low-cost fabrication is required..$^{15,16}$ In recent years, field-effect transistor (FET)-based sensors have received much attention owing to their unique properties such as low power requirements for operation, precision, low cost due to well-established chip manufacturing processes, label-free character, and ease of surface functionalization. ${ }^{10,17,18}$ Bio-FETs consist of a bio-recognition layer and transducer, and have become promising candidates in point-of-care testing (POCT) applications due to development in state-of-the-art fabrication techniques. ${ }^{19}$

A FET consists of a metal oxide and a semiconductor channel, with three electrodes, namely source, drain, and gate, ${ }^{20}$ as will be detailed later. Bio-FET sensors are conventionally based on novel functional materials such as metal-oxide-semiconductors including zinc oxide $(\mathrm{ZnO}),{ }^{21,22}$ and indium gallium zinc oxide (IGZO), ${ }^{23}$ organic semiconductors including poly(3,4-ethylene dioxythiophene) (PEDOT) ${ }^{24-28}$ one-dimensional (1D) nanostructured materials including silicon nanowires $(\mathrm{SiNWs})^{29-32}$ and carbon nanotubes (CNTs), ${ }^{33,34}$ and two-dimensional (2D) nanostructured materials such as graphene ${ }^{35}$ transition metal dichalcogenides (TMDCs), ${ }^{36,37}$ and black phosphorous. ${ }^{38-40}$ Nanostructured materials have been extensively used as a channel materials for FET sensors and are a better replacement for conventional metal-oxide semiconductor 
(MOS)-based devices due to high surface-to-volume ratio, unique electrical properties, high sensitivity, good chemical stability, and biocompatibility, ${ }^{41}$ making them suitable for detecting biomolecules such as neurotransmitters, proteins, nucleic acids, and cancer biomarkers. ${ }^{8,42}$ A representation of Bio-FET-based detection using nanomaterials is illustrated in Fig. 1.

Significant research has been carried out in the field of nano Bio-FETs such as a SiNW-based FET sensor for sensitive $\left(10^{-15}\right)$ dopamine (DA) detection, a PEDOT: PSS (polystyrene sulfonate) Bio-FET for DA detection in the range of $5 \mathrm{nM},{ }^{43}$ and a graphene-based FET (G-FET) with a detection limit in the range of $1 \mathrm{nM}$ with high selectivity. ${ }^{44}$ Several reviews have been reported on FET-based sensors in recent years ${ }^{45-48}$ with a focus on the materials employed, structure of the FET, and signal enhancement. However, only a few reviews on Bio-FET devices have been reported. This review aims to analyse the development of nanostructured materialbased Bio-FET sensors over the past decade. Initially, the basic structure, function, and working principle of FETbased sensors will be discussed followed by the discussion of recent developments in the detection of various analytes such as DA, glucose, nucleic acids, and cancer biomarkers. Finally, an outlook on the challenges and future perspectives will be presented, keeping in mind the potential of nanomaterials, the development of tools for synthesis/fabrication, analysis and characterization, and the prospects of devicelevel fabrication and integration for different applications.

\section{Basic Structure and Working Principle}

A biosensor is an analytical device that can estimate the amount of analyte present through the production of electrical, ${ }^{49}$ thermal, ${ }^{50}$ or optical signals, ${ }^{7,51}$ as shown in Fig. 2. A typical biosensor has three elements, (1) a bio-recognition component which can detect or recognize a biological molecule (enzymes, proteins, antibodies, tissues), (2) a transducer that converts the interaction between the bio-recognition component and the analyte into a signal, and (3) an amplifier which intensifies the signal from the transducer, ${ }^{1,52,53}$ as indicated in Fig. 3.

A FET is a semiconductor device that consists of a metal oxide semiconductor as the transaction medium. A typical FET has three electrodes, namely source, drain, and gate. ${ }^{20,54}$ It is composed of a semiconducting material with two doped regions, viz., source and drains which are separated by a channel layer (region depleted of charge carriers) under a metal gate and insulator. Generally, a FET has bottom-gate and top-gate geometries depending on the position of the source-drain electrodes and gate electrodes such as top gate (top contact), bottom gate (back channel-etch), bottom gate (coplanar) and bottom gate (etch stopper). ${ }^{20,55}$ In the case of a Bio-FET, the metal gate/insulator interface accommodates an immobilized biological sensing membrane. The semiconducting channel is in contact with electrolyte solution through the gate electrode. Generally, the insulator layer consists of $\mathrm{a} \mathrm{SiO}_{2} / \mathrm{Al}_{2} \mathrm{O}_{3} / \mathrm{Si}_{3} \mathrm{~N}_{4}$ layer. ${ }^{56}$ In most cases, highly doped silicon acts as substrate and gate electrode. ${ }^{57}$ When a potential is applied, the carrier concentration changes in the channel layer through a mechanism, such as accumulation, depletion, or inversion, which induces band bending. ${ }^{58}$ When the applied gate voltage is higher than the threshold voltage, it inverts the channel and allows current flow, thereby turning on the transistor. ${ }^{18,19}$ In a Bio-FET, the ion concentration or $\mathrm{pH}$ value of the analyte solution affects the channel conductivity. The electrostatic behaviour at the electrode-electrolyte interface changes when an analyte binds to a bio-receptor on the immobilized membrane and is enhanced through the resulting charge transfer or electrostatic gating. ${ }^{32,59-61}$ This makes the current-voltage (I-V)

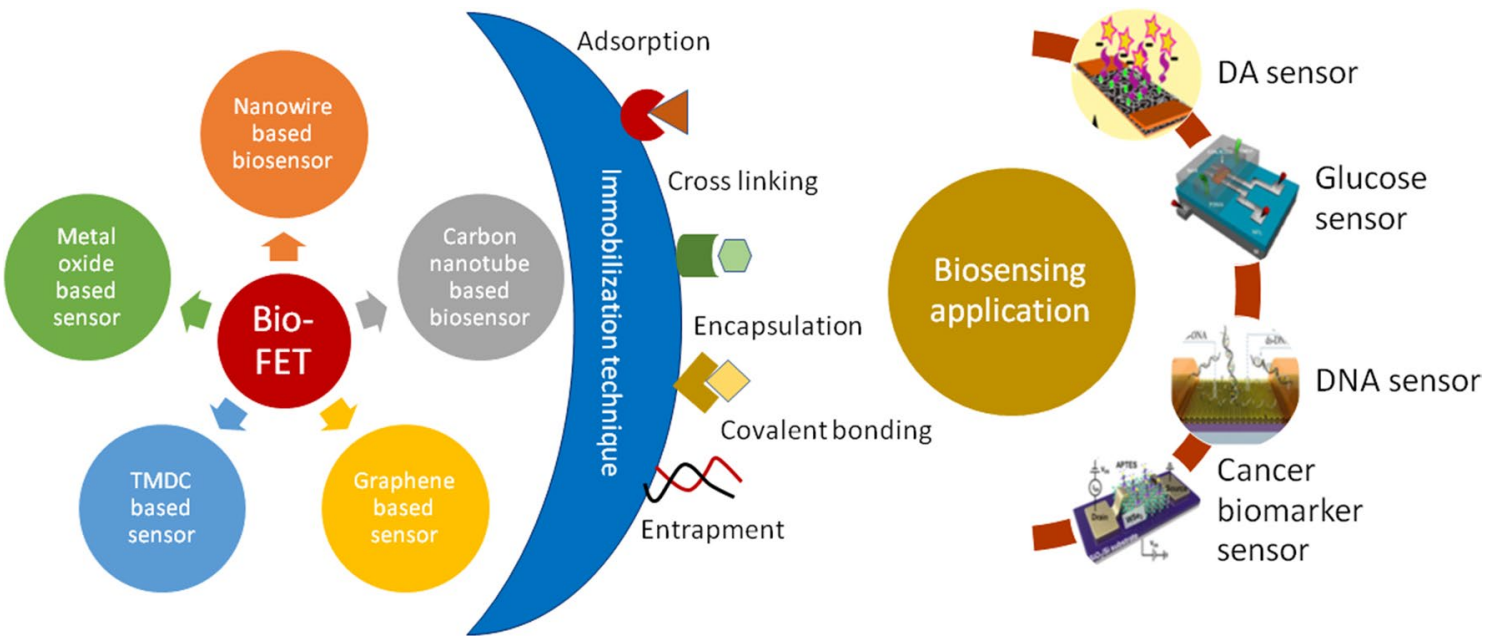

Fig. 1 Schematic illustration of Bio-FETs employing nanomaterials, their immobilization techniques and applications. 


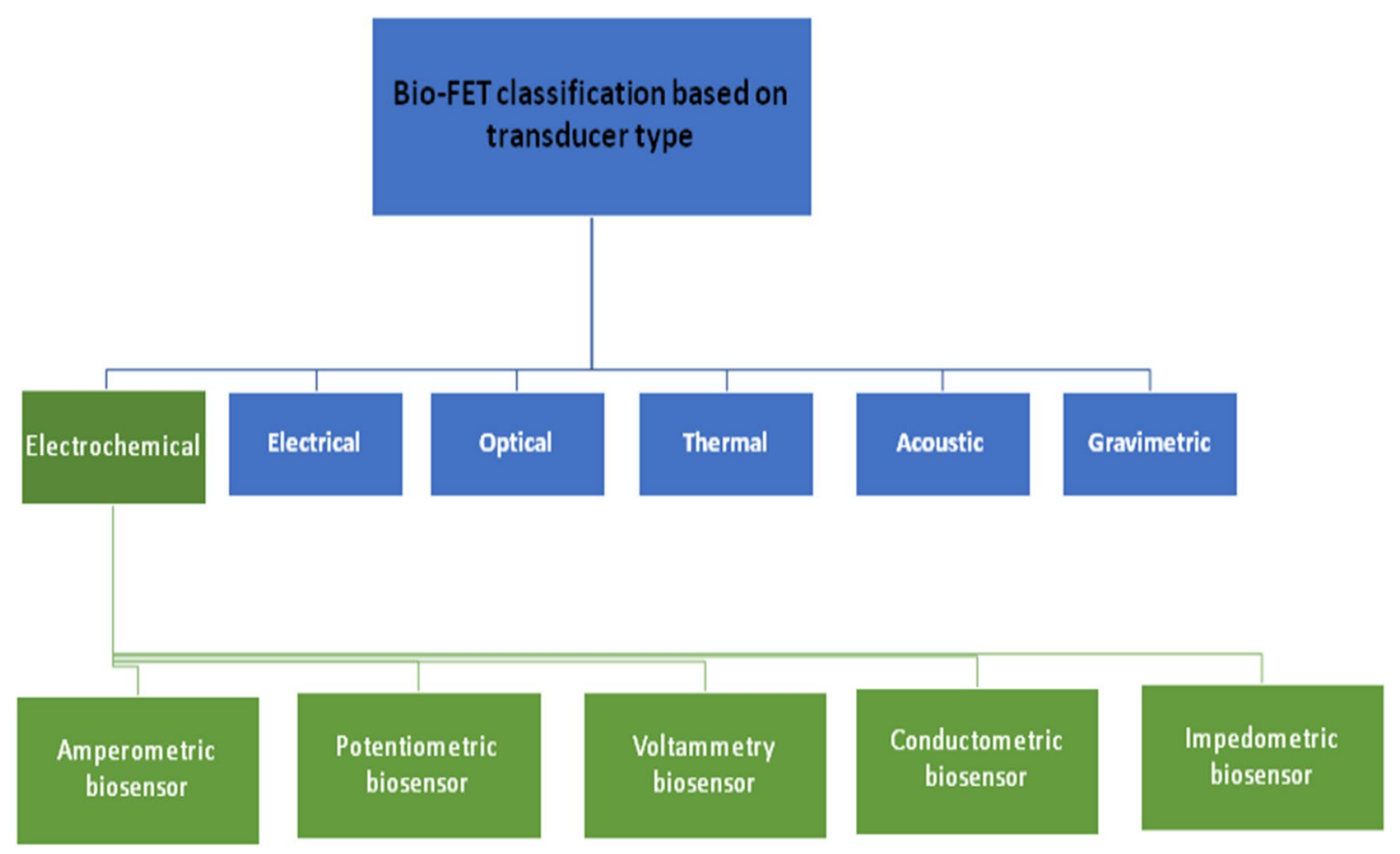

Fig. 2 Classification of biosensors based on transduction type.
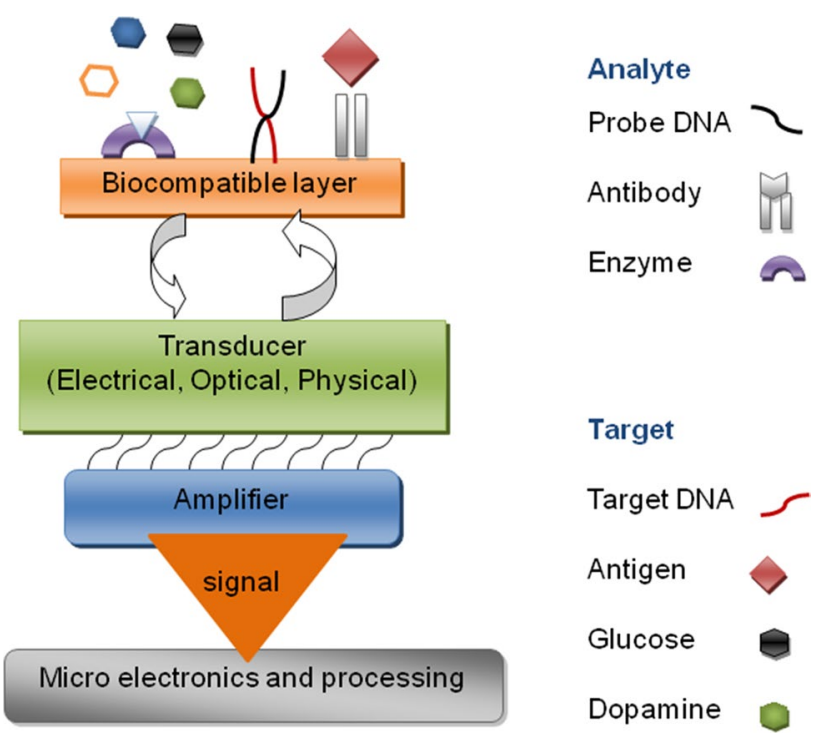

Fig. 3 Schematic representation of the working of a biosensor. An example of DNA detection is shown.

characteristics shift positively or negatively, which can be read and calibrated against known concentrations. The schematic representation of a typical laboratory-scale study employing nanomaterial Bio-FETs is provided in Fig. 4, covering stages from fabrication, testing, analysis, and final optimizations.
The response of a Bio-FET depends on the charge exchange characteristics between the analyte and the sensing membrane. When a negatively charged analyte is at a $p$-type channel, the valence band bends upwards towards the Fermi level and causes holes to accumulate at the interface of the insulator. In this case, the mobility of the majority charge carriers across the junction is enhanced. Conversely, if a positively charged analyte is placed at a $p$-type channel, the valence band bends downwards, and depletion of majority charge carriers occurs in the channel, reducing the charge transport across the junction, ${ }^{62,63}$ as shown in Fig. 5. The analyte interacts with the channel material either through physisorption or chemisorption. ${ }^{4,64}$ The binding forces and hence the sensing signal are affected by parameters such as temperature, $\mathrm{pH}$, ionic strength, and concentration of the analyte. For consistent response, interactions through chemisorption are preferred, which includes a covalent attachment, cross-linking, and entrapment. The commonly involved probe groups immobilized on the sensing membrane for Bio-FETs include the thiol group $(-\mathrm{SH})$, amino group $\left(-\mathrm{NH}_{2}\right)$, and the carboxylic acid group $(-\mathrm{COOH}){ }^{7}$ For instance, the thiol group is used as a linker to gold nanoparticles (Au NPs) due to strong Au-S bonding. On these self-assembled monolayers, the NPs are adsorbed, inducing rapid electron transfer between the substrate and Au NPs.

The sensitivity of a Bio-FET can be summarized using the following equation: 
(a)

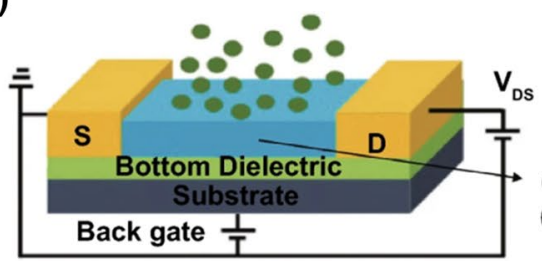

(b)

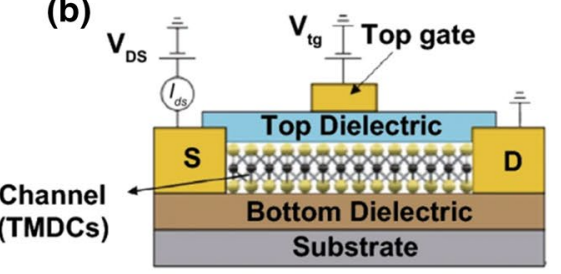

(c)

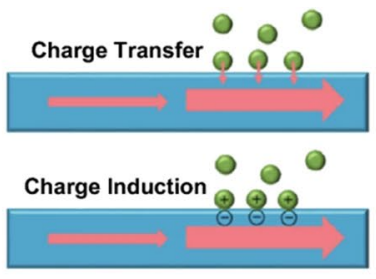

$\Longrightarrow$ Channel $\bigcirc$ Analyte Molecules $\Longrightarrow$ FET transfer process

(f)

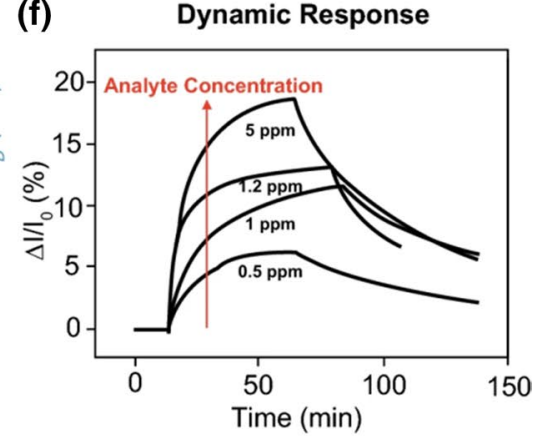

Dynamic Response (d)

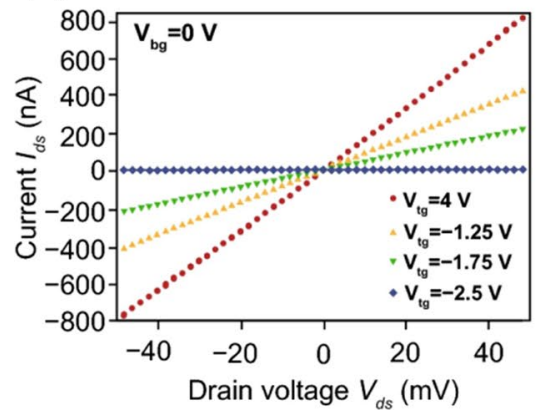

(e)

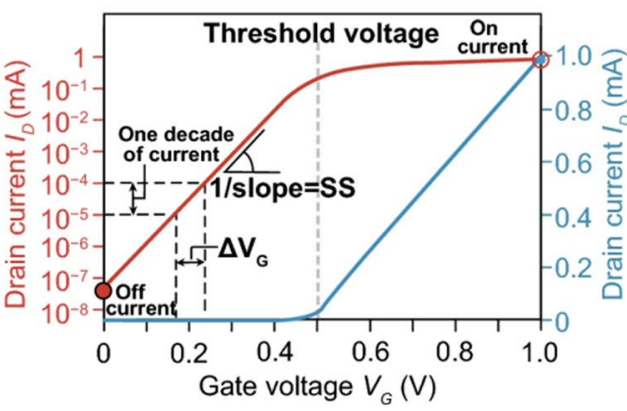

Fig. 4 (a) Structure, (b, c) working principle, (d, e) electrical characterization, and (f) sensing response of TMDC-based FET sensors. Reprinted from Chen et al. ${ }^{61}$ under the terms of Creative Commons (CC BY 4.0) license.

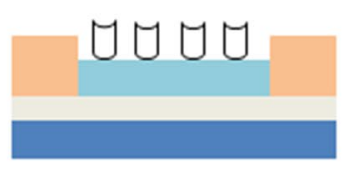

P-type channel

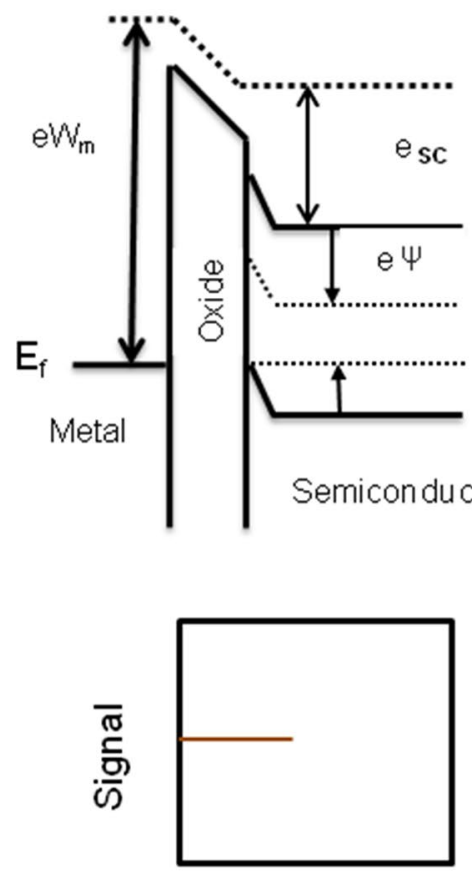

Time

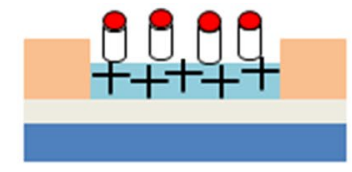

Positive charge accumulation
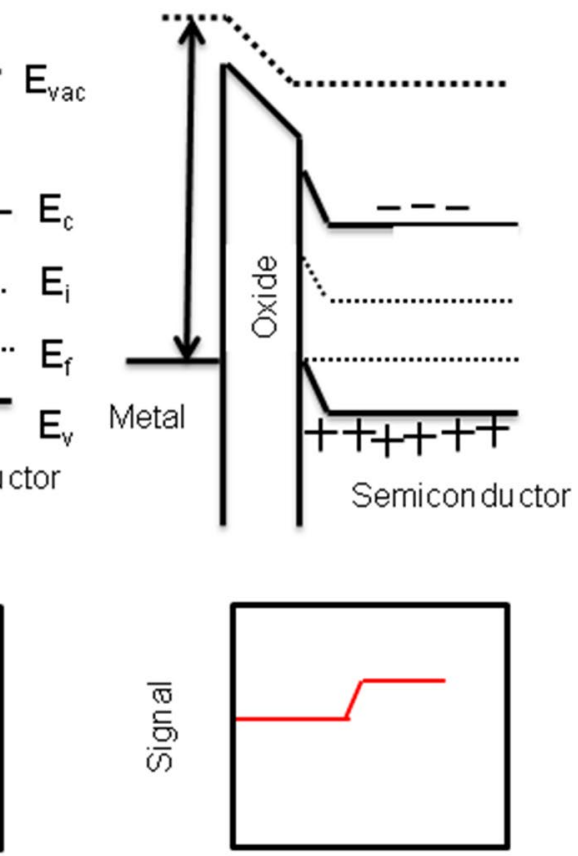

Time

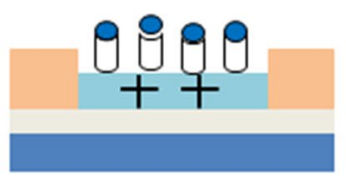

Negative ch arge accumulation

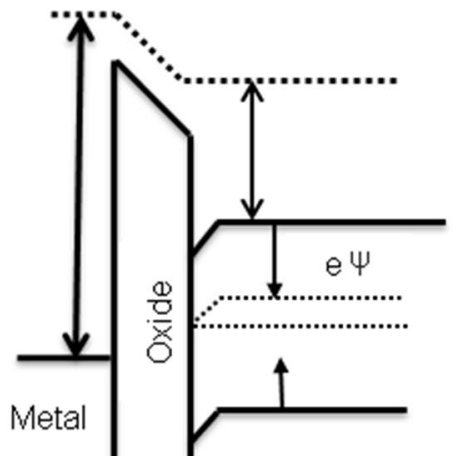

Semiconductor

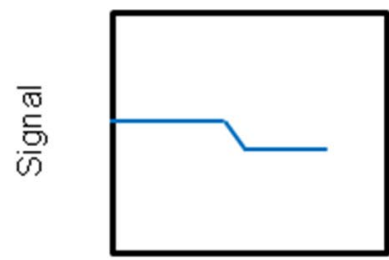

Time

Fig. 5 Working principle of a $p$-type channel biosensor. (a) Charge accumulation on the surface. (b) Energy band diagrams of a metal oxide semiconductor junction. 
$\frac{d I_{D}}{I_{D}}=\left(\mathrm{dc} \frac{d \rho}{d c}\right)\left(\frac{d v_{\mathrm{EG}}}{d \rho}\right)\left(\frac{d I_{D}}{d v_{\mathrm{EG}}} \frac{1}{I_{D}}\right)$

\section{(Sensitivity) (i) (ii) (iii)}

where (i) is the charge exchange at the sensor interface, quantified by the change in the drain-source current $(d \rho$, (ii) is change in effective gate voltage ( $d V_{\mathrm{EG}}$ ), and (iii) is the change in drain current $\left(d I_{\mathrm{D}}\right)$. Here, $d c$ represents the change in analyte concentration, and $I_{D}$ represents the change in the current without the target analyte (from a baseline exposure).

\section{Nanomaterials as a Sensing Platform for Bio-FETs}

In recent years, Bio-FETs have been extensively used for real-time label-free detection of biomolecules. The sensing performance of a Bio-FET being influenced predominantly by the channel material, many researchers have explored several options, from inorganic to organic materials, complimentary biomolecules, macroscopic to $1 \mathrm{D} / 2 \mathrm{D}$ materials, conducting polymers, and so on for a wide range of analytes, including gases, ions, organics, and biomolecules. Nanomaterials exhibit unique and tunable electrical and optical properties, including high specific surface area, excellent mechanical properties, good thermal and chemical stability, high catalytic activities, and usually a facile synthesis process, which all facilitate their furthering for high-performance biosensing. ${ }^{65,66}$ Nanostructure-based Bio-FETs have shown a very low limit of detection (LOD) towards specific biomolecular systems. Various nanomaterials such as metal oxides, ${ }^{67}$ graphene, ${ }^{68}$ and TMDC ${ }^{69}$ have been explored and reported for FET biosensors, as they can be used for flexible biosensor fabrication with high selectivity and sensitivity. The defining characteristics of a nanomaterial-based BioFET are shown in Fig. 6, detailing the parameters that can be helpful for direct comparisons between different materials as well as for establishing benchmark performance. BioFETs with 1D nanomaterials such as SiNWs and CNTs have shown high sensitivity, for example in DA detection down to the femtomolar range (fM), as well as selective detection of single virus particles using a SiNW-FET. ${ }^{70}$ However, 1D nanomaterials such as SiNW-based FET sensors are still limited in their practical applications due to relatively higher cost in large-scale fabrication compared with G-FET sensors and lack device scalability due to difficulties in precise orientation (which considerably influences charge transfer and hence the sensing performance). ${ }^{71}$

In the following sections, we highlight some important biosensors developed using semiconductors and layered

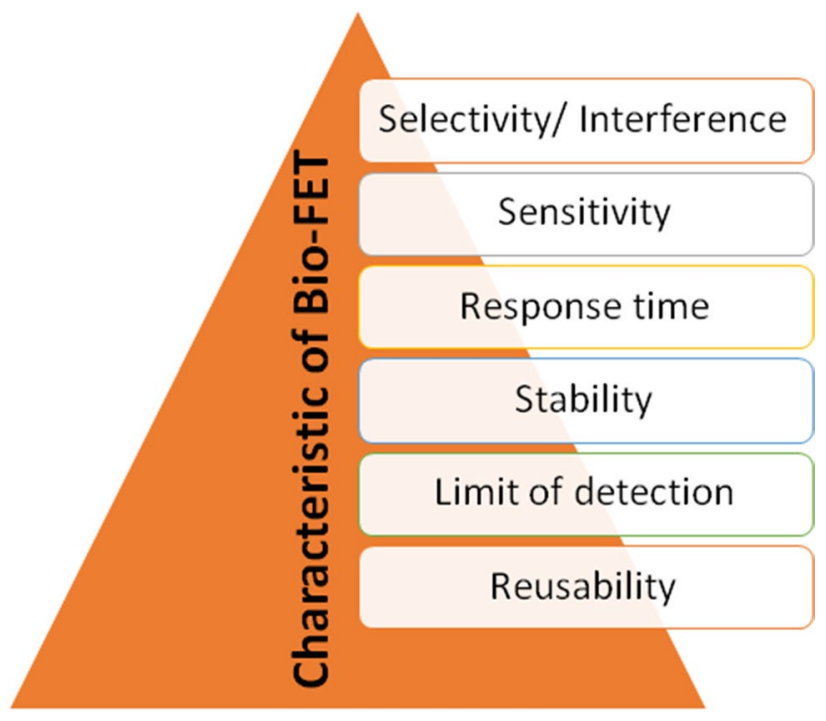

Fig. 6 Characteristics of Bio-FET sensors which help define benchmarks and comparative analyses between materials, device structures, and analytical practices.

nanomaterials. Some of the significant results will be discussed in detail.

\section{Detection of DA}

DA is a significant neurotransmitter in the human body that plays a vital role in the functioning of the central nervous system, hormone regulation, and the cardiovascular system. ${ }^{72-74}$ The normal dopamine level in the human body is in the range of $10^{-6} \mathrm{M}$ to $10^{-8} \mathrm{M}^{75,76}$ Abnormal levels of DA can lead to various neurological disorders such as Parkinson's disease, Alzheimer's disease, and schizophrenia. ${ }^{59,77-79}$ Therefore, rapid, portable, low-cost, and precise detection of DA levels in the human body is very important for diagnosis and disease prevention. ${ }^{80}$ Various Bio-FETbased devices have been reported for the detection of DA with high selectivity and sensitivity.

A SiNW has a large surface-to-volume ratio which enables local charge transfer on the surface of the wire that could be sensed through accumulation or depletion of charge carriers inside the SiNW-FET owing to an electric field effect. This leads to the modulation of conductance when analyte molecules specifically bind to the bio-recognition elements at the surface of the nanowire. SiNWs have been fabricated by either a bottom-up or top-down approach which typically requires metal-catalytic growth and optical or e-beam lithography. This is a high-cost technique. For instance, Lin et al.$^{81}$ developed a poly-SiNW Bio-FET for the detection of DA. The poly-SiNW channel was fabricated by poly-Si sidewall spacer technique. In this study, 
the SiNW channel was functionalized with an amino group introduced by 3-aminopropyltriethoxysilane (APTES) and then cross-linked to carboxyphenyl boronic acid (CPBA). The selective binding of CPBA towards dopamine forms a dopamine-boronate ester complex, producing a net negative charge at the poly-SiNW-FET interface and a change in the drain current. This device showed sensitivity in the range of $10^{-15} \mathrm{M}$. The sensing mechanism is schematically represented in Fig. 7.

Recently, a deoxyribonucleic acid (DNA)-aptamer-modified multiple parallel-connected (MPC) SiNW-FET for selective detection of DA was reported by Li et al. ${ }^{82}$ The MPC SiNW-FET device is composed of hundreds of $p$-type single crystals, while traditional SiNW-FETs possess only a single or a few SiNWs. These multiple connections of SiNW enable high detection sensitivity, larger transconductance, and better signal-to-noise ratio when compared to traditional SiNW-FETs. The aptamer is a smaller molecule relative to other bio-receptors (enzyme or antibodies), and therefore binding of DA is closer to the transducer channel, and the immobilization density of the aptamer is higher, resulting in a stronger electrical signal and hence relatively higher sensitivity, evident from the detection of DA down to $10^{-11}$ M. Even though the SiNW-FET showed excellent sensitivity towards DA, which may be attributed to its small size and high surface-to-volume ratio, it still suffers from selectivity which is not explained.
In addition to SiNWs, both single-walled (SWCNT) and multiwalled CNTs have also been explored in the construction of the Bio-FET channel. ${ }^{83}$ The rolling-up direction of the graphene sheets and the alignment of the $\pi$-orbitals determine the electronic properties of the nanotubes. ${ }^{84}$ Dharmalingam et al ${ }^{85}$ stated that the large specific surface area of CNTs facilitates the immobilization of a large number of functional molecules at the surface. The pure and high-quality SWCNT has been prepared by postsynthesis treatment, including ultracentrifugation, electrophoresis, chromatography, or post-synthesis selective etching. But it is very difficult to control surface defects and contamination that lead to low carrier mobility of the FET device. An alternative approach was proposed by $\mathrm{Li}$ et al. ${ }^{86}$ to synthesize high-quality semiconducting SWCNTs (S-SWCNT) via in situ selective hydrogen etching. The S-SWCNT integrated FET could detect DA down to $10^{-18} \mathrm{~mol} / \mathrm{L}$ in phosphate-buffered saline (PBS) solution. In an electrolyte gate FET (EG-FET), the semiconducting channel is directly in contact with a gate electrode through an electrolyte. In an EG-FET, the channel conductance is altered by the gate voltage through the capacitive fieldeffect mechanism at the channel/electrolyte interface. An EG-FET can modulate conductance at an ultralow voltage due to high parallel plate capacitance. Similarly, Bhatt et al. ${ }^{87}$ successfully demonstrated a low-cost and flexible polyimide substrate for sensitive detection of DA below

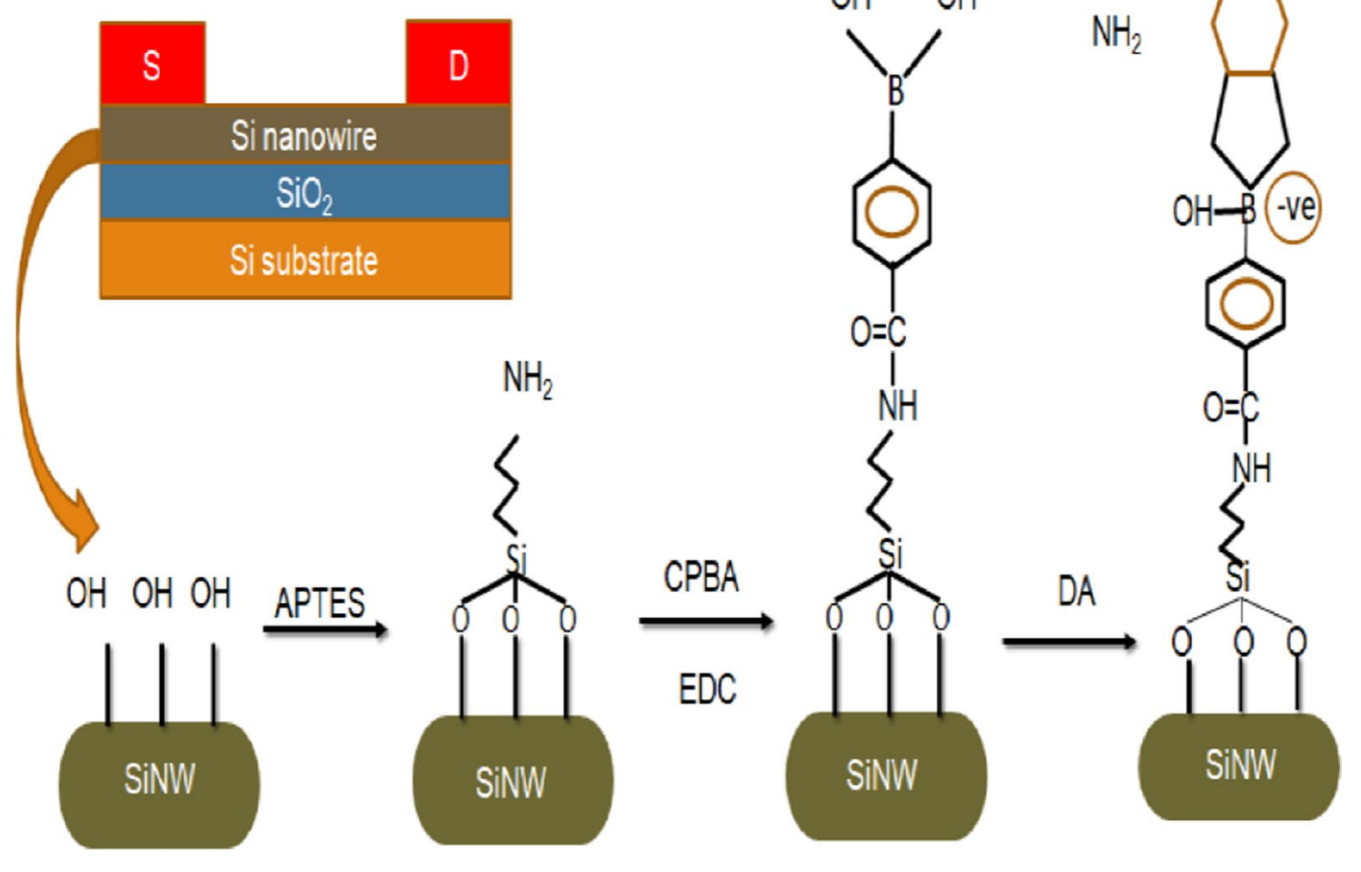

Fig. 7 A SiNW Bio-FET for DA detection. A one-dimensional nanostructure offers benefits of high sensitivity and ease of functionalization, albeit having drawbacks of a tedious fabrication process and poor repeatability. 
femtomolar (fM) range using an electrolyte gating CNTFET. The amide group of the polyimide substrate covalently functionalizes on its surface, which eliminates the need for additional APTES functionalization.

Joshi et al. ${ }^{88}$ made a highly sensitive regenerative and nonenzymatic dopamine sensor using CNTs on an interdigitated electrode, with the source and drain formed by $\mathrm{Cr} / \mathrm{Au}$. The selective formation and charge transfer through a boronate ester modulates the charge on the semiconducting channel. The detection range of dopamine was observed from $0.1 \mu \mathrm{M}$ to $1 \mathrm{fM}$ in $10 \mathrm{mM}$ PBS solution with the CNT-FET device being regenerated in hydrochloric acid. In addition, the performance of the biosensor was also found to be influenced by the $\mathrm{pH}$, ionic strength, and nature and chemical structure of the buffer solution. The same group also demonstrated dopamine-based CNT-FET sensors with varying concentration of DA in three different buffer solutions, namely MES (2-(N-morpholino) ethanesulfonic acid), HEPES (4-(2-hydroxyethyl) piperazine1-ethane sulfonic acid), and TRIS (tris(hydroxymethyl) aminomethane) and observed varying sensor responses. The different concentrations of DA solutions prepared in the MES buffer showed an increase in drain current as the concentration of DA increased, similar to the behaviour observed in the PBS buffer. However, the drain current decreased with the same variations in DA concentrations in HEPES and TRIS buffer solutions, the difference being attributed to the chemical structure of the buffer solution. The -OH group of the HEPES and TRIS forms a boronate ester complex with CPBA instead of CPBA and DA, interfering with and lowering the electrical response of the sensor.

Since the invention of graphene in 2004, it has thrived as a channel material in FET sensors due to exceptional electronic properties such as carrier mobility $\left(15,000 \mathrm{~cm}^{2} \mathrm{~V}^{-1}\right.$ $\left.\mathrm{s}^{-1}\right)$ at room temperature, high surface area $\left(2630 \mathrm{~m}^{2} \mathrm{~g}^{-1}\right)$, electrical conductivity, easy chemical functionalization, high flexibility, ambipolar nature, and biocompatibility. ${ }^{84,89-93}$ The atomic thickness of graphene allows low-noise operation, ${ }^{94}$ and the aspect ratio (width/length) greatly influences the transconductance $\left(g_{\mathrm{m}}\right)$ and particularly the noise. ${ }^{95}$ Noise is generated due to the random movement of electrons in the trap states. A high $g_{\mathrm{m}}$ value and large surface area reduce the flicker noise, which complicates the surface conduction phenomena of MOS (caused by carrier recombination and interface traps). ${ }^{96,97}$ Graphene and other 2D materials are free of dangling bonds, which reduces the scattering centres and interface traps. ${ }^{59,98}$ For semiconductors, flicker noise is mostly generated between the gate oxide and the silicon substrate. This is due to surface trapping states and releasing current carriers. The flicker noise of graphene is suppressed owing to the charge traps at the interface. These traps act as external scattering centres.
The flicker noise (1/f) is due to the fluctuation of the number of defects or gate oxide trap states and is defined by the equation

$S=\frac{q^{2} N_{o t} W L}{f}$

where $S$ is the power spectral density of current noise, $q$ is the elementary charge, $N_{\mathrm{ot}}$ is the density of oxide traps, $W$ is the width of the channel, $L$ is the length of the channel, and $f$ is the frequency. ${ }^{99}$

A solution-gated graphene transistor (SGGT) can exhibit high sensitivity, high throughput detection, and is suitable for real-time application. This device can also operate at low voltage (less than $1 \mathrm{~V}$ ) in an aqueous medium, which is a requirement for biological sensing. In an SGGT, the gate insulator is replaced by an electrolyte that is directly in contact with the graphene channel. The selectivity of the SGGT device is further improved by functionalizing the gate electrode with a biocompatible layer. Zhang et al. ${ }^{44}$ proposed an SGGT Bio-FET sensor for DA detection, able to detect DA with a limit of $1 \mathrm{nM}$. Mechanistically, $p$ doping upon the addition adsorption of DA at the graphene channel through strong $\pi-\pi$ stacking interactions was proposed as the source of the signal change. The sensitivity of a G-FET towards DA detection had a limitation of $1 \mathrm{nM}$. DA was oxidized to ortho-dopamine quinone at the gate electrode at a suitably applied potential, leading to a potential drop over the electric double-layer capacitance (EDLC) $\left(C_{\mathrm{G}-\mathrm{E}}\right)$ and consequently to an increase in the potential drop over the $\operatorname{EDLC}\left(C_{\mathrm{E}-\mathrm{C}}\right)$ where $C_{\mathrm{G}-\mathrm{E}}$ and $C_{\mathrm{E}-\mathrm{C}}$ were the capacitance of the gate/electrolyte and electrolyte/channel interfaces, respectively. An increased selectivity towards DA was achieved by modifying the graphene gate with Nafion, wherein interfering molecules like ascorbic acid (AA) and uric acid (UA) gave a signal which was 3-4 orders of magnitude lower than that of DA. The negative charge of the Nafion in PBS solution can electrostatically interact with negatively charged species such as UA and AA. Due to this interaction, the interference substance could effectively distinguish from DA. The device achieved a detection limit of $1 \mu \mathrm{M}$ for AA and $10 \mu \mathrm{M}$ for UA. Freeman et al. ${ }^{100}$ analysed dopamine by using a chemically modified ion-sensitive FET (ISFET) with a detection limit of $7 \times 10^{-5} \mathrm{M}$. The ISFET was functionalized with phenylboronic acid with the formation of a boronate-dopamine complex on the surface of the channel. A timeline of the evolution of nanomaterial-based Bio-FETs for the detection of DA is presented in Fig. 8.

Metal-oxide-semiconductors have been recently incorporated into Bio-FETs owing to their good electrical properties, including high electron mobility, transparency, and easy fabrication. ${ }^{101}$ Nanostructured metal oxides (NMO) provide improved electron-transfer kinetics and strong adsorption 


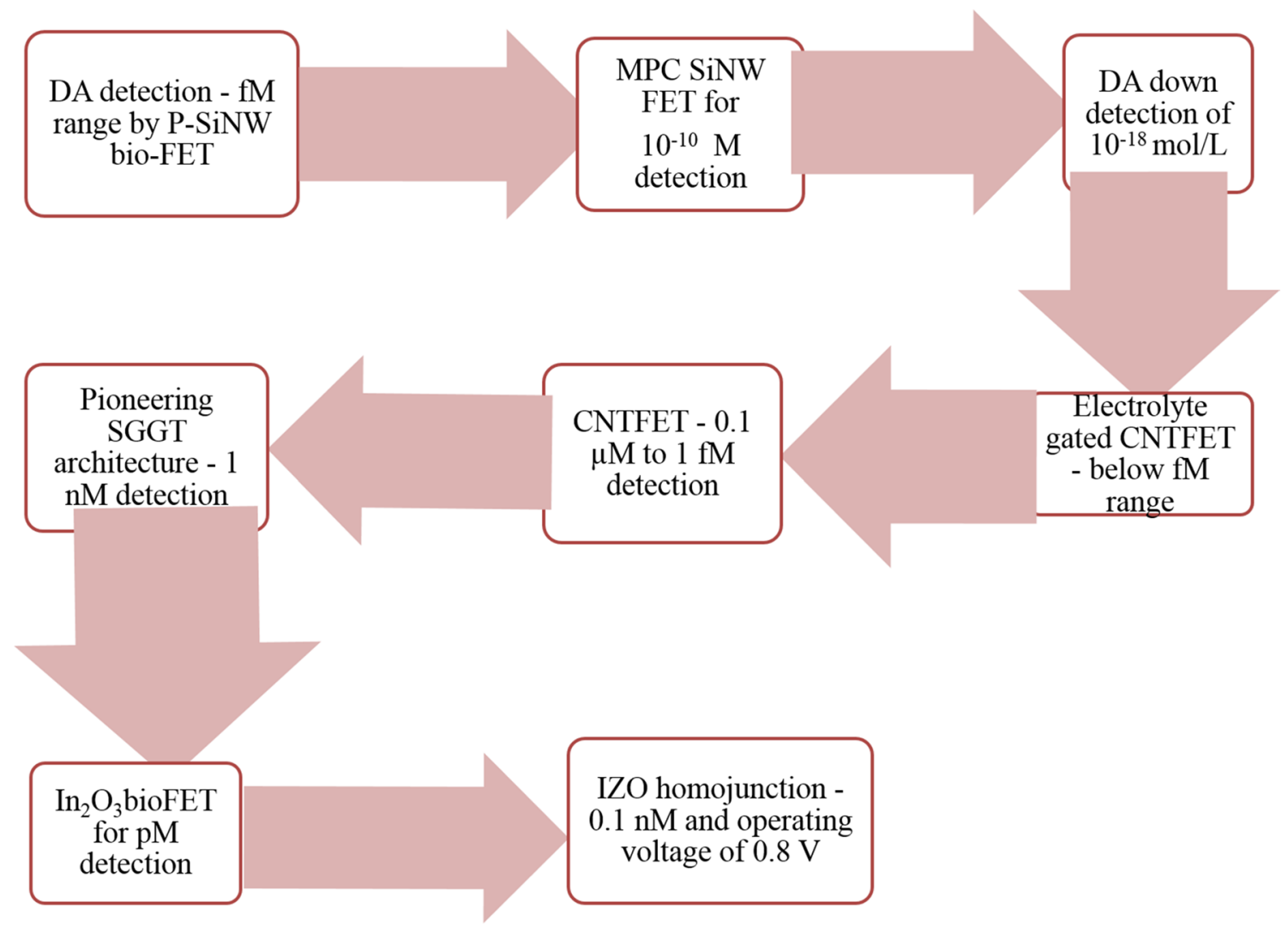

Fig. 8 A timeline of the events and findings of nanomaterial-based Bio-FETs for the detection of DA.

of biomolecules. ${ }^{17}$ The target biomolecules can be immobilized on the surface of NMOs through physical adsorption or covalent bonding. $\mathrm{ZnO}, \mathrm{In}_{2} \mathrm{O}_{3}, \mathrm{Cu}_{2} \mathrm{O}$, and IGZO are some of the examples of NMO-based biosensors for the detection of various analytes. Generally, metal-oxide-semiconductors are realized via a solution process or a sputtering process. Kim et al. ${ }^{102}$ produced highly sensitive Bio-FET arrays, fabricated using chemical lift-off lithography. $\operatorname{In}_{2} \mathrm{O}_{3}$ was used as the active semiconducting channel material, and DA selectively bound to an aptamer immobilized on it, which changed the charge potential on the ultrasensitive $\operatorname{In}_{2} \mathrm{O}_{3}$ surface. The sensor could detect a picomolar concentration of dopamine and showed good selectivity.

Recently, transistors based on ion gels and solid electrolytes have become increasingly suitable for device-level applications, as they can function at very low voltages because of strong ionic/electronic interaction with electrolytes. ${ }^{103}$ The accumulation of charge carrier density at the gate/electrolyte and electrolyte/semiconductor channel interfaces is induced by strong EDLC formation. When the gate voltage is applied in such devices, the ions in the electrolyte mobilize to the gate/electrolyte and semiconductor/ electrolyte interfaces. This leads to the formation of EDLC with huge capacitance. Due to the strong electrostatic gating effect, net charge carriers accumulated or are depleted in the semiconductor channel. Thus, the high capacitance facilitates the EDLC with high carrier density with a low gate voltage. ${ }^{104}$ Liu et al. ${ }^{105}$ proposed an EDL transistor based on an oxide homo-junction for DA detection with an operating voltage of $0.8 \mathrm{~V}$. The sensing mechanism was the modification of the surface potential of the indium tin oxide (ITO) bottom gate electrode induced by the selective binding of DA molecules to phenylboronic acid, with high sensitivity (up to $0.1 \mathrm{nM}$ ) and good selectivity. The EDL transistor was fabricated on an ITO glass substrate, and solid electrolyte and IZO were deposited on it. The ITO bottom gate was surface-functionalized and immobilized by APTES and CPBA. When a voltage was applied to the gate electrode, strong EDL effects were observed on the electrolyte/IZO channel interface. Because of the EDL effect, a large specific capacitance of $-2.0 \mu \mathrm{F} / \mathrm{cm}^{2}$ was obtained at $0.1 \mathrm{~Hz}$.

\section{Bio-FETs for DA Detection: A Summary}

The concentration of DA is extremely low $\left(<10^{-10} \mathrm{M}\right)$ in the extracellular fluid of Parkinson's disease patients and in the urine/blood of paragangliomas patients, dictating the minimal levels that need to be detected. However, the 
existing electrochemical techniques mostly reach limits at around $10^{-9} \mathrm{M}$. Moreover, the redox potential of DA in urine, blood, and the central nervous system coexists with other electroactive species such as AA and UA, posing barriers for constructing high-performance Bio-FETs with high selectivity. In this regard, Bio-FETs based on $\mathrm{SiNW}^{81}$ and MPC-SiNW ${ }^{82}$ have been developed to detect DA, offering pathways for selective detection. Even though studies with poly-SiNWs have demonstrated sensitivities in the $10^{-15} \mathrm{M}$ range, and aptamer-modified SiNW-FETs in the $10^{-10} \mathrm{M}$ range towards DA, they have not been shown to be selective, and have slow response times. The whole SGGT device ${ }^{44}$ has been developed specifically to improve selectivity towards DA by modifying the gate electrode with the biocompatible polymer Nafion, with a sensing capability down to $1 \mathrm{nM}$. However, the whole device is required to be immersed in an aqueous electrolyte, which will degrade the device stability due to unwanted side reactions.

A device that can operate with minimal voltage requirements is essential for portable applications. This has been demonstrated by the EG-FET device, which lowers voltage requirements by strong EDL modulations at the electrode/ electrolyte channel interface. An IZO-based Bio-FET ${ }^{105}$ is another potential combination for operating with low gate voltages $(0.8 \mathrm{~V})$, also due to the strong EDL effect at the channel/electrolyte interface. Thus, a strong electrostatic interaction at the analyte/material interface is crucial for the achievement of low-power-consumption Bio-FETs for DA detection.

Although FET-based DA biosensors have become competitive for POCT applications, an important challenge in the commercial biosensor market is the cost, which is typically high due to the active channel material (example being indium) and/or the fabrication process. Specifically, nanoparticle morphologies (such as 1D nanomaterials) that have been proven to be highly sensitive for DA detection often require processes such as chemical vapour deposition (CVD) and lithography techniques, for steps such as material deposition and growth, transfer to the substrate, patterning, and further processing steps. The main drawbacks of this procedure are a requirement of special instruments in a clean room with a well-controlled environment, expensive chemicals for processing, skilled personnel, and the associated low yields of workable devices.

In addition to sensitivity and selectivity, flexible and stretchable Bio-FET devices can immensely benefit the field of wearable diagnostic devices and implantable electronic chips. Flexible polymeric substrates are gaining attention in POCT diagnostics, as they are thin, lightweight, easy to use, and biocompatible. A CNT EG-FET, for example, has been fabricated on a flexible polyimide substrate.

Reusability of the device is an alternate approach to reduce the cost of the device. Indeed, the CNT-FET device ${ }^{88}$ with high sensitivity down to the fM range can be regenerated in an acidic environment, enabling the reusability of the sensor device. With progress in the field of wearable and paintable electronic devices, it stands that this progressing field can be of critical importance to the field of DA detection using nanomaterials (see Table I).

\section{Detection of Glucose}

Diabetes mellitus is a group of metabolic disorders that are a worldwide health problem. This disorder is caused by the deficiency of insulin in blood glucose, being responsible for heart disease, kidney failure, and blindness. Therefore, continuous glucose monitoring is essential for early diagnosis as well as management of diabetes. ${ }^{106,107}$ The development of sensitive and selective glucose sensors with a recent focus on developing wearable, non-invasive, and reliable devices is a thrust area of recent research. ${ }^{108,109}$ Glucose detection can be done through different methods such as electrochemical, fluorescent, acoustic, transdermal, and optical sensing techniques. ${ }^{14,110}$ However, FET-based biosensors have promising potential in glucose detection with the advantages of sub-micromolar detection, developments in miniaturization

Table I Summary of DA detection using nanomaterials

\begin{tabular}{llllll}
\hline Material & Receptor & Detection limit & Fabrication technique(s) & Substrate & References \\
\hline Graphene & CPBA & $1 \mathrm{nM}$ & Thermal evaporation, CVD & Glass/PET & 44 \\
P-SiNW & CPBA & $1 \mathrm{fM}$ & $\mathrm{P}-$ Si side-wall spacer technique & Silicon & 81 \\
MPC-SiNW & APTAMER & $10^{-11} \mathrm{M}$ & $\mathrm{CVD}$, thermal evaporation, photolithography & Silicon & 82 \\
$\mathrm{SWCNT}$ & $\mathrm{CPBA}$ & $10^{-18} \mathrm{~mol} / \mathrm{L}$ & In situ hydrogen etching & PET & 86 \\
$\mathrm{CNT}$ & $\mathrm{CPBA}$ & $10 \mathrm{fM}$ & Photolithography, thermal evaporation, spray deposition & Polyimide & 87 \\
$\mathrm{CNT}$ & $\mathrm{CPBA}$ & $1 \mathrm{fM}$ & Photolithography, thermal evaporation, spray deposition & Polyimide & 88 \\
$\mathrm{Al}_{2} \mathrm{O}_{3}$ gate/silicon & $\mathrm{CPBA}$ & $7 \times 10^{-5} \mathrm{M}$ & Chemical modification & Silicon & 100 \\
$\mathrm{In}_{2} \mathrm{O}_{3}$ & $\mathrm{CPBA}$ & $100 \mathrm{pM}$ & Sol-gel processing, spin coating, chemical lift-off lithography & Silicon & 102 \\
$\mathrm{IZO}$ & $\mathrm{CPBA}$ & $0.1 \mathrm{nM}$ & Plasma-enhanced CVD (PECVD), sputtering & Glass & 105 \\
\hline
\end{tabular}


and cost of the sensing chip, and low-power operation, to mention a few. ${ }^{111}$

The mechanism of glucose detection is based on the oxidation of glucose to gluconic acid and hydrogen peroxide in the presence of glucose oxidase $\left(\mathrm{GO}_{\mathrm{x}}\right)$, as shown in Fig. 9. The subsequent dissociation of $\mathrm{H}_{2} \mathrm{O}_{2}$ to oxygen leads to potential changes in the sensing membrane, which are detected as follows: ${ }^{25,28}$

Glucose $+\mathrm{O}_{2}+\mathrm{GO}_{\mathrm{x}}$ gluconic acid $+\mathrm{H}_{2-} \mathrm{O}_{2}$

$\mathrm{H}_{2} \mathrm{O}_{2} \mathrm{O}_{2}+2 \mathrm{H}^{+}+2 \mathrm{e}^{-}$

Besteman et al. ${ }^{112}$ introduced glucose sensors based on SWCNTs in the FET configuration. The major challenge in employing CNTs is the separation of metallic nanotubes from semiconducting ones and the fact that they are too small to interface with larger biomolecules. Lee et al. ${ }^{33}$ investigated the fabrication of transparent and flexible SWCNT ISFET sensors for glucose detection using lithography and layer-by-layer (LbL) self-assembly. SWCNTs and poly-(diallyl dimethylammonium chloride) (PDDA) were deposited between the source and drain electrodes patterned on a flexible PET substrate. This polymer substrate is biocompatible, inexpensive, and eliminates the need for an insulator layer. Glucose has been detected by local $\mathrm{pH}$ change with the help of the $\mathrm{GO}_{\mathrm{x}}$ multilayer, with a sensitivity of $18-45 \mu \mathrm{A} / \mathrm{mM}$ in the range of $2-10 \mathrm{mM}$ of glucose.

Huang et al. ${ }^{113}$ developed real-time SGGT biosensors based on chemical vapour deposition (CVD)-grown macrographene films on a quartz substrate. Source and drain electrodes were prepared by silver paint at the edges of the graphene film. Glucose or glutamate were detected by surface-functionalized $\mathrm{GO}_{\mathrm{x}}$ with a detection limit of $0.1 \mathrm{mM}$. The increment of channel current of the SGGT device is due to the products from the oxidative reaction of glucose, while the mechanism was not investigated in detail. Kwak et al. ${ }^{114}$ fabricated an SGGT biosensor prepared by CVD-grown graphene on a flexible PET substrate. Source and drain electrodes were prepared by silver paint with a binding epoxy at the two ends of the graphene film. The sensor could detect glucose levels in the range of 3.3-10.9 mM, at par with the physiological range for diabetes screening. In the case of tuning charge carriers, the electrochemical gate is more preferred than the back gate contact because the applied gate potential in the solution-gated Bio-FET interacts directly with biomolecules and double-layer formation at the graphene/solution interface. However, the interaction between $\mathrm{H}_{2} \mathrm{O}_{2}$ and $\mathrm{GO}_{\mathrm{x}}$ is not explained clearly, and the LOD of the device is high, while other transistor-based glucose sensors could detect glucose down to $\mu \mathrm{M}$ levels. To explore a novel Bio-FET and achieve better sensing performance, we need to understand the mechanism of SGGT-based glucose sensors. Zhang et al. ${ }^{115}$ demonstrated a new type of glucose sensor based on an SGGT in which both channel and gate are made up of graphene. In this device, graphene gate electrodes were functionalized with Pt nanoparticles, leading to a much lower detection limit of $30 \mathrm{nM}$. Khadija et al. ${ }^{116}$ demonstrated a nonenzymatic glucose sensor where graphite oxide (GO) was the channel material and was assembled between the electrodes. The sensitivity of the device was enhanced by adding $\mathrm{Ag}$ and $\mathrm{Cu}$ nanoparticles. A positive gate voltage increased the density of conducting electrons in the channel, enhancing conductivity and consequently the drain current. GO with $\mathrm{Cu}$ nanoparticles showed better performance at low glucose concentration, with the detection limit at $1 \mu \mathrm{M}$, albeit a wide range of detection from 1 $\mu \mathrm{M}$ to $30 \mathrm{mM}$.

Ultrathin (few atoms) TMDCs have been found to exhibit excellent gate electrostatics, high surface-to-volume ratio, tunability in bandgap and electronic properties with layer thickness, scalability down to monolayer dimension, and surface activities when used as channel materials, ${ }^{117-120}$ with

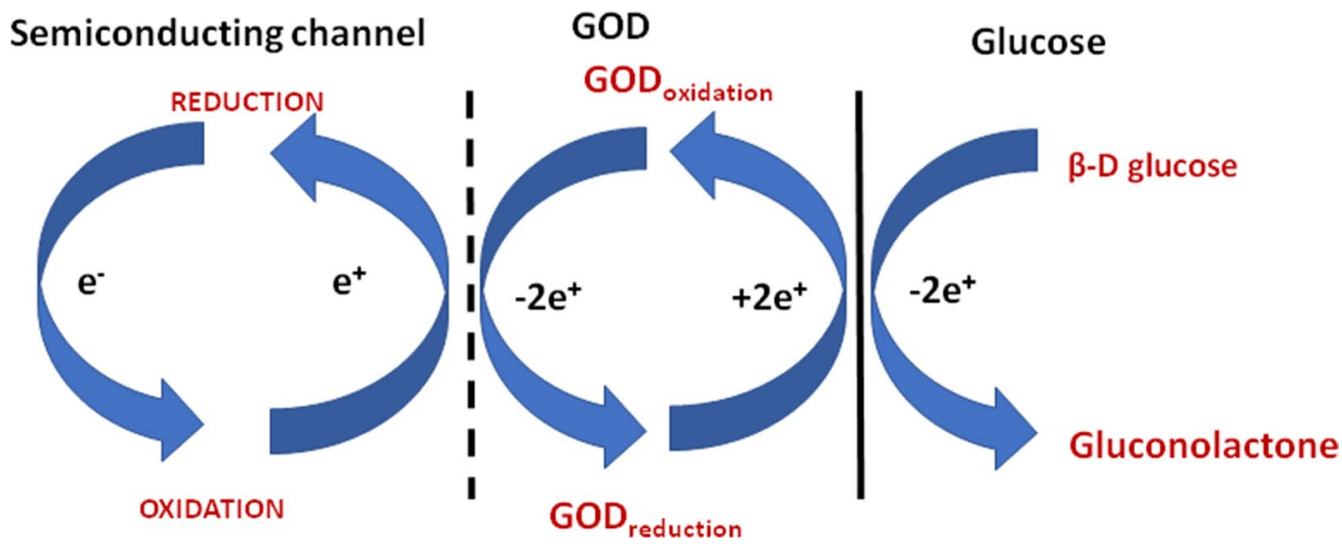

Fig. 9 Schematic representation of reaction mechanism between the Bio-FET channel, glucose, and $\mathrm{GO}_{\mathrm{x}}$. 
Bio-FETs based on TMDC channels offering excellent sensitivity at room temperature for the detection of analytes. ${ }^{36,121}$ Lee et al. ${ }^{122}$ made a highly sensitive and reusable membraneless $\mathrm{WSe}_{2}$ biosensor for glucose detection, as shown in Fig. 10. They intentionally created surface defects by lowintensity $\mathrm{O}_{2}$ plasma treatment on the $\mathrm{WSe}_{2}$ channel, providing extra binding sites for holding the APTES as a linker for enhancing the sensitivity of the device. The $\mathrm{WSe}_{2}$ channel was additionally functionalized with glutaraldehyde (middle linker) and $\mathrm{GO}_{\mathrm{x}}$ (bio-receptor). The negatively charged $\mathrm{NH}_{2}$ group of APTES repelled the electrons at the APTES/WSe ${ }_{2}$ channel interface, causing $n$ doping. The redox reactions (1) and (2) produce a greater number of electrons as the concentration of glucose increases on the conductive $\mathrm{WSe}_{2}$ surface of the device, increasing the channel current, with a detection range of $1 \mathrm{mM}$ to $10 \mathrm{mM}$ of glucose.

Shan et al. ${ }^{123}$ demonstrated a $\mathrm{MoS}_{2}$-based Bio-FET for glucose detection at extremely low concentrations. $\mathrm{MoS}_{2}$ is bound through weak van der Waals interactions ${ }^{124,125}$ and has great potential as a channel material owing to a few uncoordinated bonds on the surface, a stable structure, and low leakage currents due to a direct bandgap. ${ }^{120,126} \mathrm{MoS}_{2}$ nanosheets showed good electrochemical sensitivity to glucose with a linear response in the range of $0-30 \mathrm{mM}$ with a detection limit in the range of $300 \mathrm{nM}$. The FET device had a sensitivity of $260.75 \mathrm{~mA} \mathrm{mM}^{-1}$ and a response time of $<1 s$.

Recently, ZnO-based FET biosensors have been widely investigated in glucose detection owing to their high isoelectric point (IEP) of -9.0 which facilitates immobilization of enzymes such as $\mathrm{GO}_{\mathrm{x}}$ and cholesterol oxidase. ${ }^{21,127,128}$ They are also attractive because of their biocompatibility, nontoxicity, chemical stability, direct bandgap $(3.37 \mathrm{eV})$, and maturity of high-yield synthesis methods. ${ }^{127,129-131}$ Among the various morphologies of nanostructured $\mathrm{ZnO}$, nanorods (NRs) have been widely used for biomolecule immobilization. ${ }^{95}$ By using $\mathrm{ZnO}$ nanowires functionalized with $\mathrm{Ag}$, Ali et al. ${ }^{127}$ demonstrated glucose detection coupled with a commercial MOSFET. Here, response time and stability of (a)

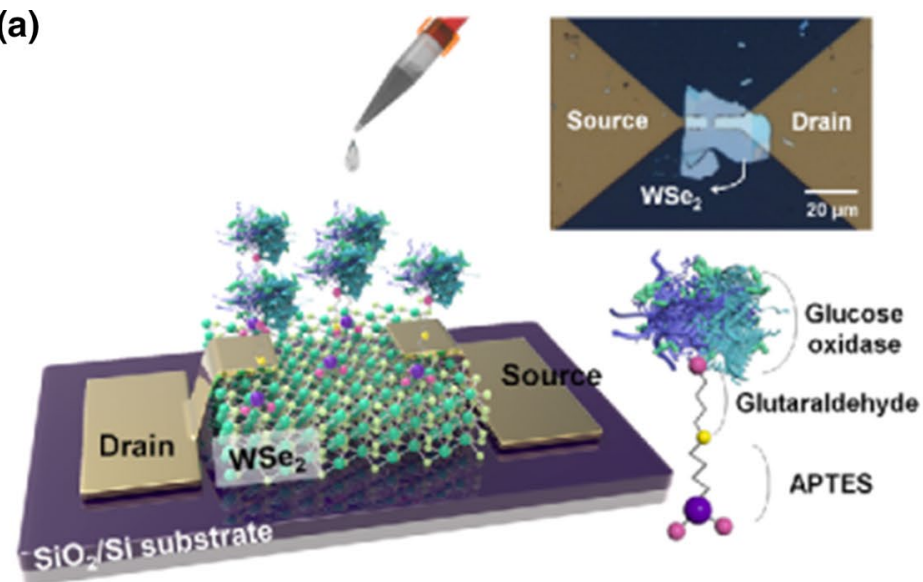

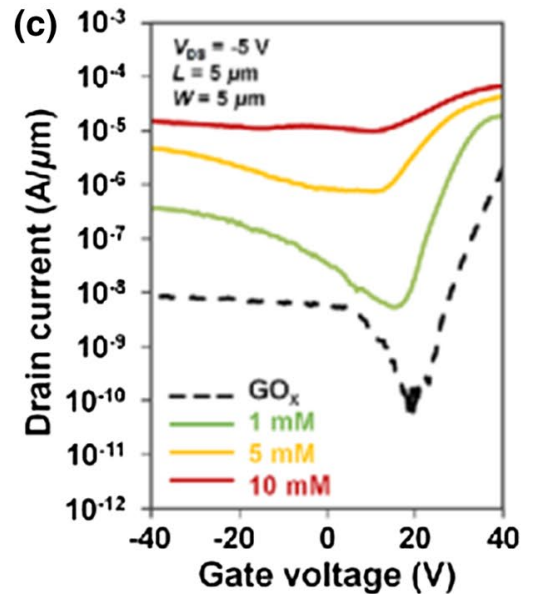

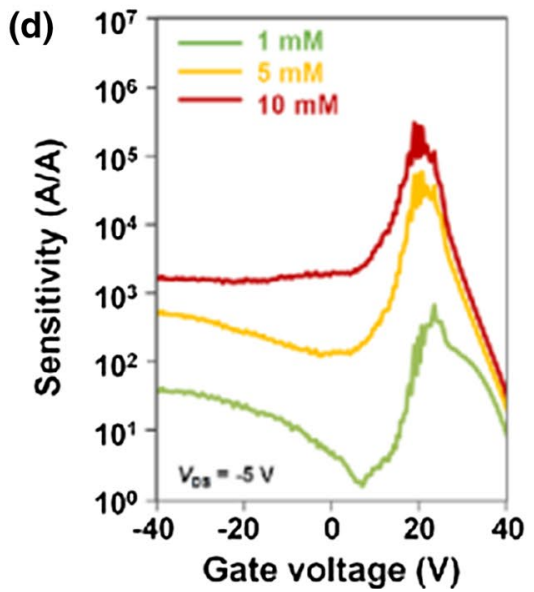

(b)

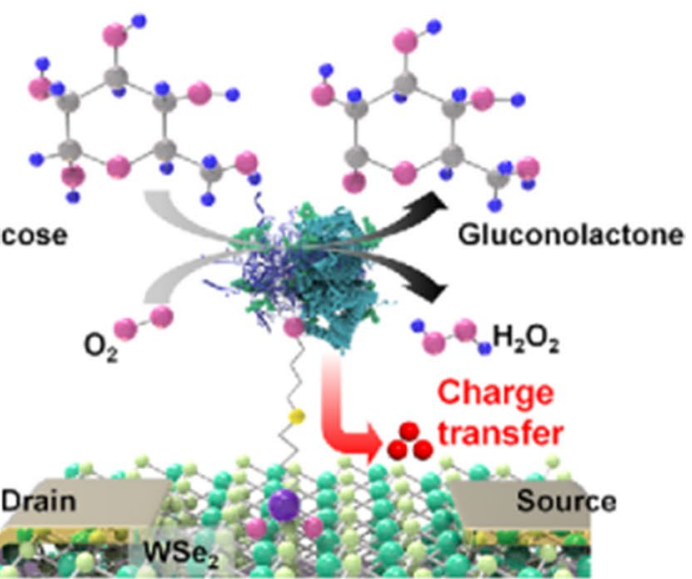

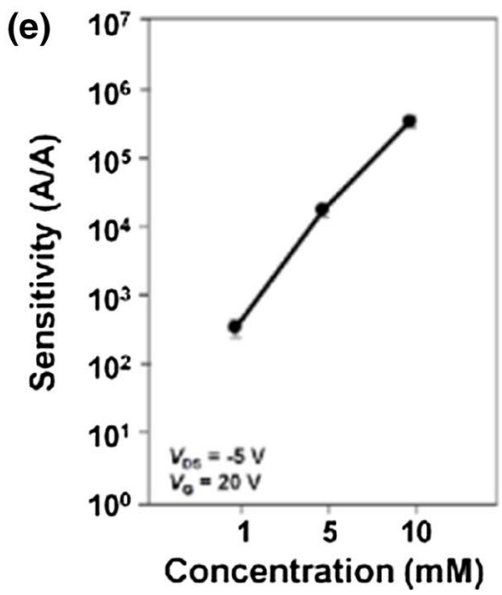

Fig. 10 The glucose sensing performance of membrane-less $\mathrm{WSe}_{2}$ Bio-FET. (a) $\mathrm{WSe}_{2}$ Bio-FET with $\mathrm{GO}_{\mathrm{x}}$ bioreceptor. (b) Electron transfer reactions to the $\mathrm{WSe}_{2}$ channel surface. (c) $\mathrm{I}_{\mathrm{D}}-\mathrm{V}_{\mathrm{G}}$ characteristics of the $\mathrm{WSe}_{2}$ Bio-FET exposed to different concentrations of glucose. (d) Sensitivity values as a function of gate voltage under different glucose concentrations. (e) Average sensitivity values from five different glucose concentrations. Reprinted from Lee et al. ${ }^{122}$ under the terms of Creative Commons (CC BY-NC-ND 4.0) license. 
the extended gate MOSFET were studied through three different $\mathrm{ZnO}$ nanowire structures, such as a vertically aligned uniform $\mathrm{ZnO}$ nanowire, a uniform nonaligned $\mathrm{ZnO}$ nanowire, and a nonuniform nonaligned $\mathrm{ZnO}$ nanowire. Among these, the uniform and well-aligned $\mathrm{ZnO}$ nanowire sensors showed stable signals and a response time less than $100 \mathrm{~ms}$. Nonaligned and nonuniform distribution of $\mathrm{ZnO}$ nanowires sensor showed slower response time. This is because nonuniform structures take more time to have contact with the analyte solution. It was concluded that the distribution and alignment of the $\mathrm{ZnO}$ nanowire partially impacts the response time and stability of the sensor design. Moreover, the $\mathrm{ZnO}$ nanowire has an IEP of 9.5, while the $\mathrm{GO}_{\mathrm{x}}$ has an IEP equal to 4.2. This huge difference leads to electrostatic interaction of the $\mathrm{GO}_{\mathrm{x}}$ on the $\mathrm{ZnO}$ nanowire surface. The immobilization of $\mathrm{GO}_{\mathrm{x}}$ on the $\mathrm{ZnO}$ nanowire is responsible for the direct electron transfer between active sites of the enzyme and electrode surface. This implies that the high sensitivity, stability, and higher affinity towards the enzyme by $\mathrm{ZnO}$ nanowires.

Though $\mathrm{ZnO}$ shows high sensitivity, it suffers from poor stability; hence, it is necessary to modify the surface to improve stability without loss of sensitivity and selectivity. To this end, copper oxide, iron oxide, magnesium oxide, and titanium oxide materials have been employed as composites. ${ }^{17}$ Iron oxide NPs enhance the surface area and improve the stability of $\mathrm{ZnO}$ NRs. ${ }^{132}$ Ahmad et al. ${ }^{133}$ have reported a nonenzymatic glucose sensor with vertically oriented $\mathrm{ZnO}$ NRs between Ag electrodes (source and drain) on a seeded substrate. The poor response was noticed with unmodified $\mathrm{ZnO}$ NRs, whereas a linear response was observed with $\mathrm{Fe}_{2} \mathrm{O}_{3}$-modified $\mathrm{ZnO}$ NRs, as shown in Fig. 11. The reaction mechanism can be given as $2 \mathrm{Fe}(\mathrm{III})+$ glucose $2 \mathrm{Fe}(\mathrm{II})+$ Gluconolactone $+\mathrm{H}_{2} \mathrm{O}$

Gluconolactone $+\mathrm{H}_{2} \mathrm{O}_{2} \mathrm{H}^{+}+$Gluconate

$2 \mathrm{Fe}(\mathrm{II}) 2 \mathrm{Fe}(\mathrm{III})+2 \mathrm{e}^{-}$

The modified $\mathrm{ZnO} \mathrm{NR} \mathrm{Fe}_{2} \mathrm{O}_{3}$ had a sensitivity of 105.75 $\mu \mathrm{A} / \mathrm{mMcm}^{2}$ in the range of $0.05-18 \mathrm{mM}$ with a detection limit of $12 \mu \mathrm{M}$ and a response time of $10 \mathrm{~s}$. Long-term storage stability was investigated for 10 weeks and found to be quantitatively better than nonenzymatic glucose sensors. Glucose detection in samples from blood and serum showed a consistent response, taking this device a step closer to field applications. Similarly, Fathollahzadeh et al. ${ }^{134}$ used ZnO NRs as an $n$-type channel layer on the surface of gold interdigitated electrodes for a liquidgated FET. The change in conductance was attributed to hydronium ions produced as the product of the enzymatic reaction between $\mathrm{GO}_{\mathrm{x}}$ and glucose. The sensitivity of the
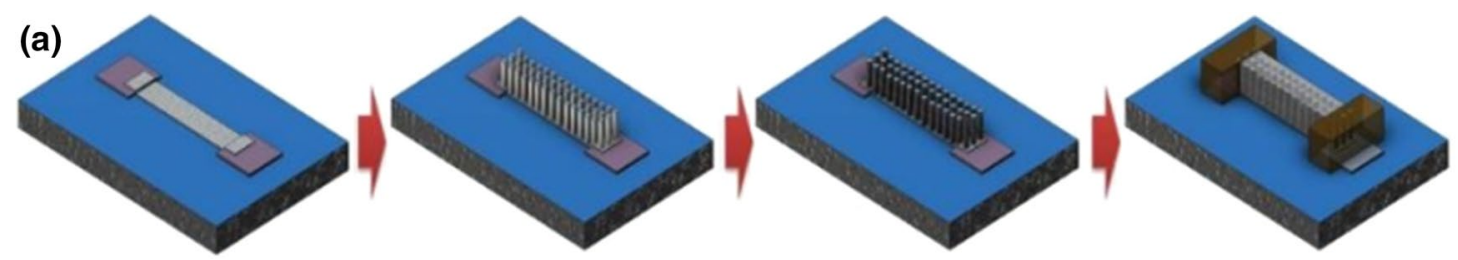

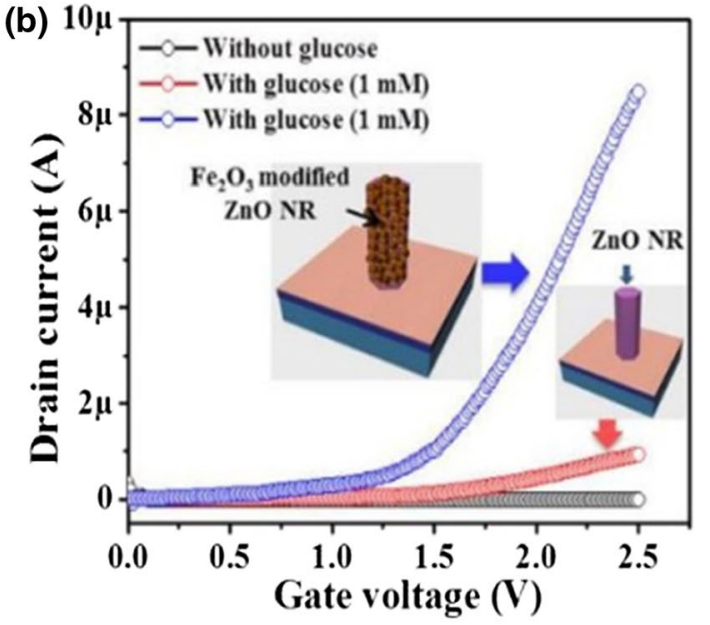

Fig. 11 (a) Schematic representation of a nonenzymatic glucose sensor based on a $\mathrm{Fe}_{2} \mathrm{O}_{3}$-ZnO NR Bio-FET. (b) Transfer characteristics of the nonenzymatic glucose sensor in $0.1 \mathrm{mM}$ PBS solution. (c)

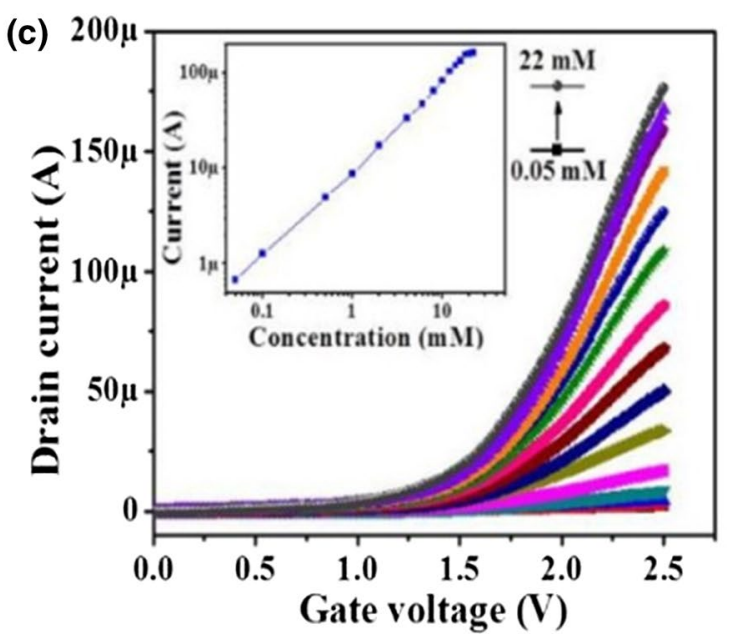

Transfer characteristics of the $\mathrm{Fe}_{2} \mathrm{O}_{3}$-ZnO NR FET for the detection of glucose in $0.1 \mathrm{mM}$ PBS. Reproduced with permission from Ahmad et al. ${ }^{133}$ Copyright (2017), Elsevier. 
device was $60.5 \mu \mathrm{AmM}^{-1} \mathrm{~cm}^{-2}$ within the range of $0-13.9$ $\mathrm{mM}$ of glucose.

Recently, Zong and Zhu ${ }^{135}$ developed a glucose biosensor using $\mathrm{ZnO}$ NRs for continuous glucose monitoring, as shown in Fig. 12. ZnO NRs were grown between two microelectrodes by an alternating current (AC) electric fieldassisted hydrothermal method, and the $\mathrm{ZnO}$ NRs transduced the biological interaction into an electrical signal at a different frequency, measured by a lock-in amplifier. $\mathrm{GO}_{\mathrm{x}}$ immobilization led to high sensitivity of $1.6 \mathrm{~mA} / \mu \mathrm{Mcm}^{2}$ within an area of $180 \mu^{2}$, and the detection limit was found to be as low as $1 \mu \mathrm{M}$. The reduced size makes minimally invasive glucose monitoring feasible, such as in dermal interstitial fluid (IF). This ZnO NR Bio-FET showed 38-hour continuous monitoring of the glucose with long-term stability and minimal drift.

Du et al. ${ }^{136}$ introduced transparent amorphous IGZO, hitherto a promising candidate in various electronic devices due to excellent electrical and optical properties and transparency. ${ }^{137}$ The IGZO FET was functionalised with amino silane groups that were cross-linked to $\mathrm{GO}_{\mathrm{x}}$. Glucose sensing occurs through the decrease in $\mathrm{pH}$ during glucose oxidation, which modulates the positive charge of the amino silane groups attached to the IGZO surface.
The change in charge affects the number of acceptor-like surface states, changing the electron density in the $n$-type IGZO semiconductor. An increase in glucose concentration causes an increase in acceptor states and a decrease in drain-source conductance due to a positive shift in the turn-on voltage. The specificity of this reaction minimized interference from the most common cross-sensitizing analytes, namely acetaminophen and ascorbic acid.

Chen et al. ${ }^{138}$ developed an ultrasensitive and highly reproducible FET biosensor using 2D metal oxide semiconductors through a solution process. The biosensor was constructed using $\mathrm{In}_{2} \mathrm{O}_{3}$ on $\mathrm{Si} / \mathrm{SiO}_{2}$ substrate by a facile spin-coating process. The quasi- $2 \mathrm{D} \operatorname{In}_{2} \mathrm{O}_{3}$ surface was functionalized with boronic acid, and the glucose-sensing performance was studied through the boronic acid-glucose interaction. The boronate anions produced as a result induces electrostatic gating and depletes the carrier concentration at the semiconductor/electrolyte interface in the $\mathrm{In}_{2} \mathrm{O}_{3}$ film. A significant change in sensing current over the concentration range of $10^{-13}$ to $10^{-3} \mathrm{M}$ was observed, with an LOD below $7 \mathrm{fM}$ and a precise sensing range from $10^{-11}$ to $10^{-5} \mathrm{M}$. Figure 13 elucidates the progress in the study of nanomaterials for the detection of glucose.
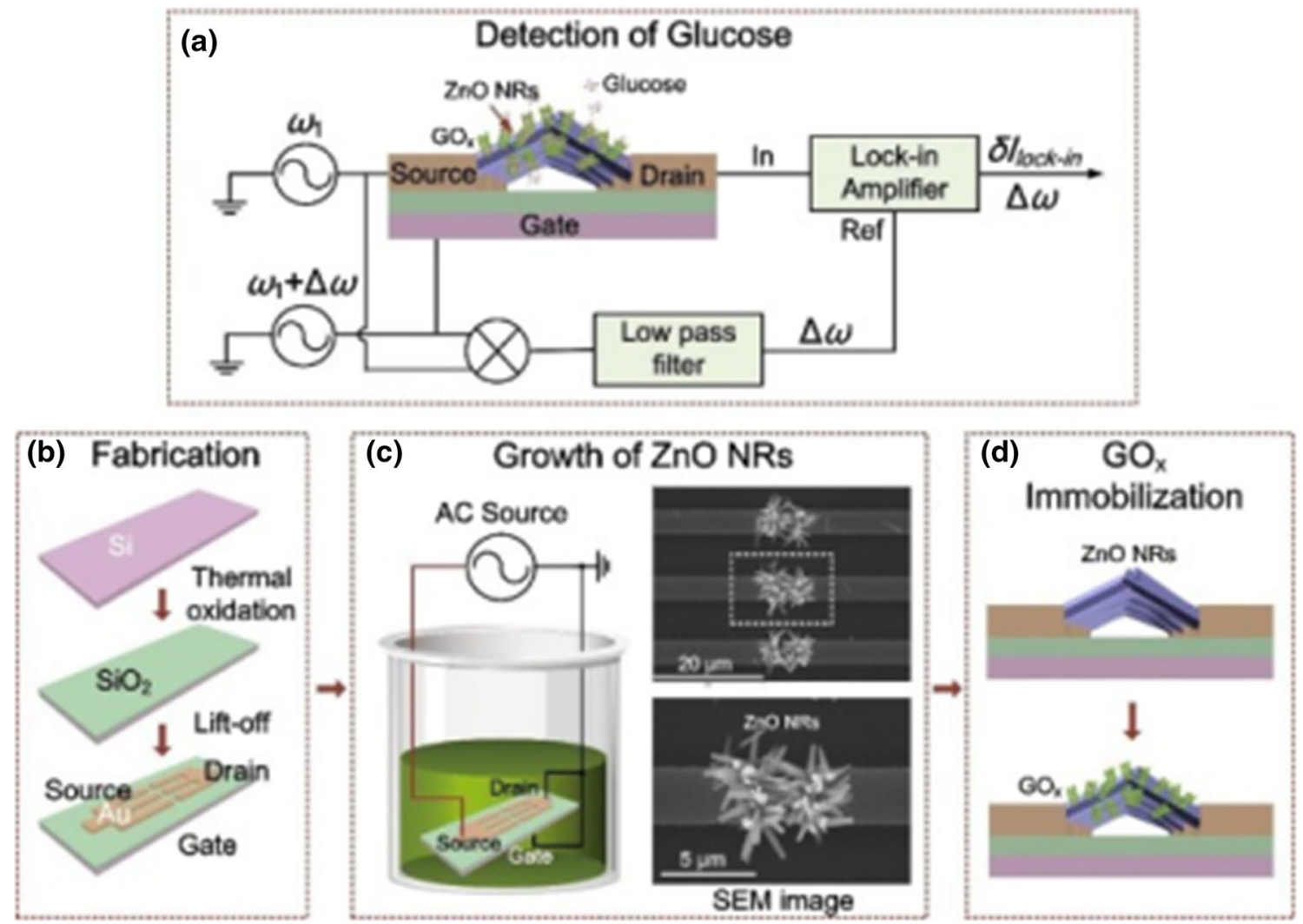

Fig. 12 (a) Configuration of a ZnO NR-based Bio-FET for detection of glucose. (b) The fabrication process, (c) growth of ZnO NRs, and (d) functionalization process of the bio-receptor. Reproduced with permission from Zong and Zhu ${ }^{135}$ Copyright (2017), Elsevier. 


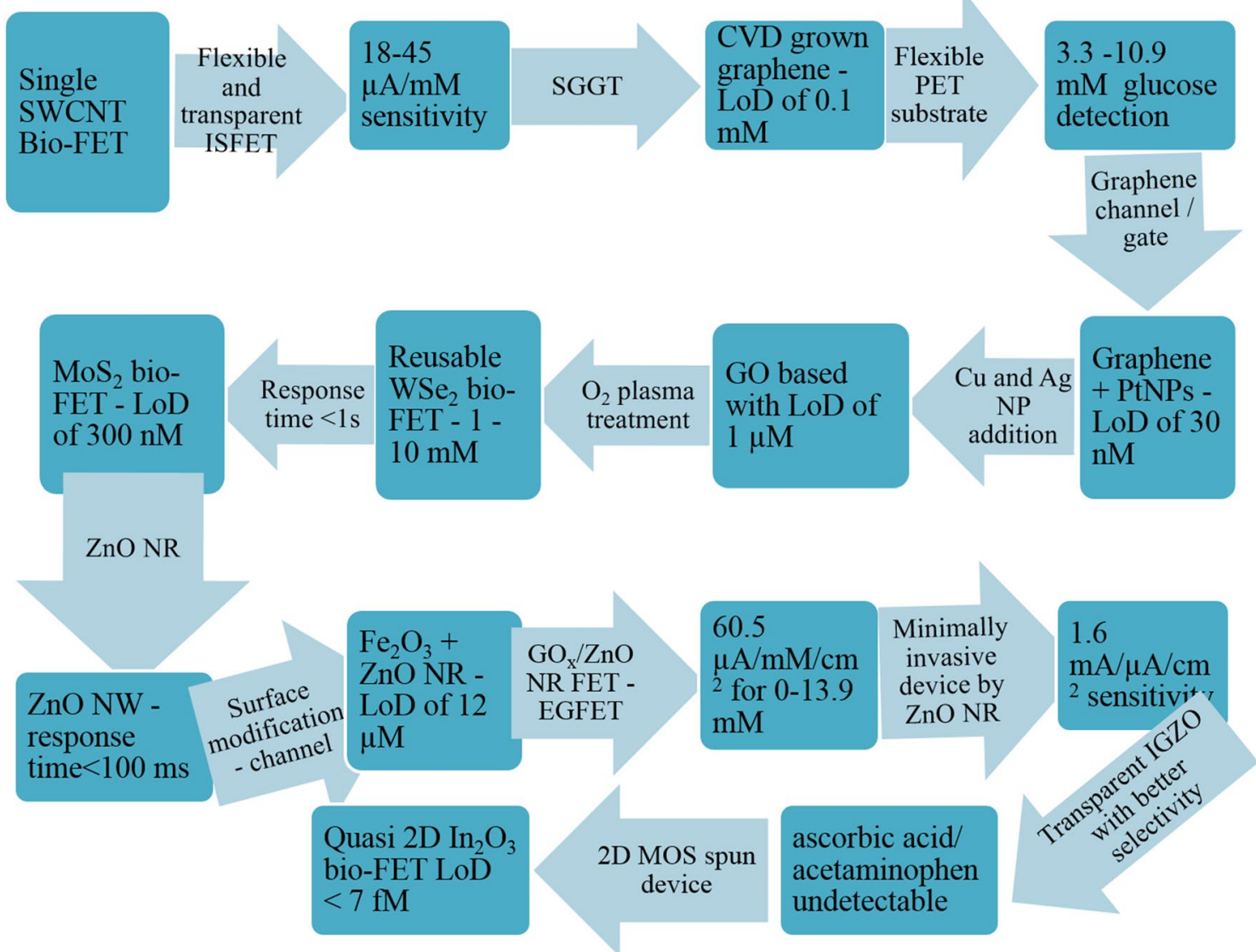

Fig. 13 Detection of glucose using nanomaterials and progress in the same since the inception of nano Bio-FETs.

\section{Outlook on Bio-FETs for Glucose Detection}

The normal physiological range of glucose concentration is $2-30 \mathrm{mM}$ in the interstitial fluid and $0.1-0.4 \mathrm{mM}$ in tear fluid. Most of the glucose sensors are enzyme-based, whose performance depends on an enzyme to catalyse the reaction. Enzyme-based electrodes are unstable, expensive, and easily degrade the device performance if enzyme functionalization deteriorates over time. To overcome these issues, nonenzymatic sensors should be developed with additional advantages of being feasible for low-cost mass production. The long-term stability of glucose Bio-FET sensors is a challenge that has seen little progress. Along with detection of glucose using various nanostructured materials such as 1D nanomaterials, 2D nanomaterials, and metal oxides, devices with the SGGT architecture have been successfully developed, operating in aqueous electrolytes at low voltages (less than $1 \mathrm{~V}$ ), making them viable candidates for portable devices.

NMOs have attracted great attention for fabricating high-performance nonenzymatic sensors with good catalytic activity in an alkaline medium. Detection of glucose with $\mathrm{GO}$ as well as with quasi-2D $\operatorname{In}_{2} \mathrm{O}_{3}$, for example, has demonstrated detection limits in the femtomolar range. Considerable onus lately has been on the development of noninvasive and disposable glucose biosensors, which can monitor blood glucose levels in interstitial fluids continuously. Current continuous glucose monitoring (CGM) devices, albeit robust, portable, atmosphere-proof, and wirelesscompatible, can benefit from improvements in a few areas. The sensor itself, for example, needs to be replaced every few days. Another area for improvement is the possibility of accommodating a self-regulating sensor which can be part of a feedback loop for revising dietary recommendations based on the input received from the sensor. Bio-FETs are especially suited in this regard, as they have demonstrated good cyclability, low sensitivity, low power requirements, and miniaturization possibilities (Tables II, III, and IV).

\section{Detection of Nucleic Acids}

Rapid and highly sensitive DNA detection is significant for the diagnosis of genetic diseases, molecular biology, and environmental monitoring. Most DNA detections depend on real-time polymerase chain reaction (RTPCR) or optical 
Table II Summary of glucose detection using various nanomaterials

\begin{tabular}{|c|c|c|c|c|c|}
\hline Material & Receptor & Detection limit & Fabrication technique(s) & Substrate & References \\
\hline SWCNT & $\mathrm{GO}_{\mathrm{x}}$ & $2 \mathrm{mM}$ & Sputtering, lithography, LbL assembly & PET polyester & 33 \\
\hline SWCNT & $\mathrm{GO}_{\mathrm{x}}$ & $0.1 \mathrm{mM}$ & CVD, e-beam lithography & Silicon & 112 \\
\hline Graphene & $\mathrm{GO}_{\mathrm{x}}$ & $0.1 \mathrm{mM}$ & CVD & Quartz & 113 \\
\hline Graphene & $\mathrm{GO}_{\mathrm{x}}$ & $3.3 \mathrm{mM}$ & CVD & PET & 114 \\
\hline Graphene & $\mathrm{GO}_{\mathrm{x}} /$ Nafion & $30 \mathrm{nM}$ & CVD & Glass & 115 \\
\hline GO & $\mathrm{GO}_{\mathrm{x}}$ & $1 \mu \mathrm{M}$ & Thermal evaporation using shadow mask, hummers method, sputtering & Silicon & 116 \\
\hline $\mathrm{WSe}_{2}$ & $\mathrm{GO}_{\mathrm{x}}$ & $1 \mathrm{mM}$ & $\begin{array}{l}\text { Mechanical exfoliation, optical lithography, e-beam evaporation, } \mathrm{O}_{2} \\
\text { plasma treatment }\end{array}$ & Silicon & 122 \\
\hline $\mathrm{MoS}_{2}$ & $\mathrm{GO}_{\mathrm{x}}$ & $300 \mathrm{nM}$ & $\begin{array}{l}\text { Mechanical exfoliation, ultraviolet (UV) photolithography, e-beam } \\
\text { evaporation }\end{array}$ & Silicon & 123 \\
\hline $\mathrm{ZnO} \mathrm{NR}$ & $\mathrm{GO}_{\mathrm{x}}$ & $12 \mu \mathrm{M}$ & Radio-frequency (RF) magnetron sputtering & Silicon & 133 \\
\hline $\mathrm{ZnO} \mathrm{NR}$ & $\mathrm{GO}_{\mathrm{x}}$ & $3.8 \mu \mathrm{M}$ & Photolithography, hydrothermal & Glass & 134 \\
\hline $\mathrm{ZnO} \mathrm{NR}$ & $\mathrm{GO}_{\mathrm{x}}$ & $1 \mu \mathrm{M}$ & Thermal oxidation & Silicon & 135 \\
\hline IGZO & $\mathrm{GO}_{\mathrm{x}}$ & $7 \mathrm{fM}$ & Thermal oxidation, e-beam evaporation & Silicon & 136 \\
\hline $\mathrm{In}_{2} \mathrm{O}_{3}$ & $\mathrm{GO}_{\mathrm{x}}$ & $7 \mathrm{fM}$ & Thermal oxidation, e-beam evaporation & Silicon & 138 \\
\hline
\end{tabular}

Table III Summary of DNA detection using various nanomaterials

\begin{tabular}{|c|c|c|c|c|c|c|}
\hline Material & Analyte & Receptor & Detection limit & Fabrication technique(s) & Substrate & References \\
\hline Graphene & DNA & Probe DNA & $0.01 \mathrm{nM}$ & Chemically modified graphene from graphene oxide & Silicon & 71 \\
\hline $\mathrm{MoS}_{2}$ & mi-RNA & Probe mi-RNA & $0.1 \mathrm{fM}$ & Drop casting, Sputtering, photolithography & Silicon & 134 \\
\hline Graphene & DNA & Probe DNA & $0.01 \mathrm{nM}$ & CVD & Silicon & 140 \\
\hline Graphene & DNA & Probe DNA & $10 \mathrm{aM}$ & CVD & $\mathrm{Cu} / \mathrm{PMMA}$ & 141 \\
\hline $\mathrm{rGO}$ & DNA & Probe DNA & $2 \mathrm{nM}$ & $\begin{array}{l}\text { Photolithography, e-beam evaporation, } \mathrm{O}_{2} \text { plasma } \\
\text { treatment }\end{array}$ & Silicon dioxide & 142 \\
\hline $\mathrm{rGO}$ & DNA & PNA probe & $100 \mathrm{fM}$ & CVD, Photolithography, e-beam evaporation & Silicon & 143 \\
\hline Graphene & DNA & Probe DNA & $10 \mathrm{fM}$ & Direct transfer technique & Silicon & 144 \\
\hline Au/graphene & mi-RNA & PNA & $10 \mathrm{fM}$ & Photolithography, e-beam evaporation & Silicon & 145 \\
\hline $\mathrm{MoS}_{2}$ & DNA & Probe DNA & $10 \mathrm{fM}$ & Shadow mask, thermal evaporation, photolithography & Silicon & 146 \\
\hline $\mathrm{Cu}^{2+} / \mathrm{MoS} 2$ & DNA & DNA probe & $10 \mathrm{nM}$ & Optical photolithography, e-beam evaporation & Silicon & 147 \\
\hline
\end{tabular}

or electrochemical transduction that conventionally requires expensive optics and detectors as well as fluorescent/electrochemical tags. ${ }^{139}$ Recently, G-FET sensors have shown potential in nucleic acid sensing with label-free detection, high sensitivity, and rapid measurement. As mentioned previously, graphene shows an ambipolar electric field so that its semiconducting behaviour can be altered by controlling the gate voltage, as shown in Fig. 14. The minimum conductance of graphene is known as the Dirac point which indicates the potential at which the number of holes and electrons are equal. The Fermi level $\left(E_{\mathrm{f}}\right)$ of pristine graphene corresponds to the Dirac point. When a biomolecule binds on the graphene surface, there is a modulation in $E_{\mathrm{f}}$ of graphene leading to a shift in the Dirac point. In $p$ doping of graphene, the Dirac point shifts to lower potential, while in $n$ doping, the Dirac point shifts to a higher potential. ${ }^{35}$ Based on this mechanism, G-FETs have been employed as a sensing platform for detecting various analytes, including DNA, micro-ribonucleic acid (miRNA), glucose, DA, and viruses, as explained in the following section.

Mohanty et al. ${ }^{71}$ developed a chemically modified G-FET sensor for studying DNA interactions, in which singlestranded DNA (ssDNA) was connected onto a chemically modified graphene surface as a probe, and the hybridization of DNA on exposure to the complementary strand led to a huge increase in graphene conductivity. In 2010, Dong et al. ${ }^{140}$ utilized a large-sized CVD-grown G-FET sensor for label-free DNA sensing. The $\mathrm{I}_{\mathrm{ds}}-\mathrm{V}_{\mathrm{g}}$ curves showed that the gate voltage at which the base current was reached (Dirac point) could be utilized to identify the doping condition of graphene. The Dirac point shift was correlated with DNA concentration, with a sensitivity of $0.01 \mathrm{nM}$ and the capability to differentiate a single base mismatch. A G-FET designed by Campos et al. ${ }^{141}$ achieved label-free detection 
Table IV Summary of cancer biomarker detection with nanomaterials

\begin{tabular}{|c|c|c|c|c|c|c|}
\hline Material & Analyte & Receptor & Detection limit & Fabrication technique(s) & Substrate & Reference \\
\hline $\mathrm{MoS}_{2}$ & Streptavidin & Biotin & $100 \mathrm{fM}$ & $\begin{array}{l}\text { Micro-mechanical exfoliation, } \\
\text { photolithography, e-beam } \\
\text { evaporation }\end{array}$ & Silicon & 59 \\
\hline Graphene & BSA & N/A & $0.3 \mathrm{nM}$ & $\begin{array}{l}\text { Micro-mechanical exfoliation, } \\
\text { Scotch tape method, e-beam } \\
\text { lithography, a lift-off process }\end{array}$ & Silicon & 149 \\
\hline Graphene & SARS-Cov-2 Virus & SARS-Cov-2 Antigen & $1 \mathrm{fM}$ & $\begin{array}{l}\text { Photolithography, reactive ion } \\
\text { etching, thermal evaporation }\end{array}$ & Silicon & 151 \\
\hline $\mathrm{rGO}$ & HER2 & HER2- monoclonal antibody & $1 \mathrm{pM}$ & $\begin{array}{l}\text { Modified Hummers method, } \\
\text { lithography, a chemical lift-off } \\
\text { process }\end{array}$ & Silicon dioxide & 152 \\
\hline $\mathrm{rGO}$ & PSA & Monoclonal-antibody & $100 \mathrm{fg} / \mathrm{ml}$ & $\begin{array}{l}\text { Thermal evaporation using metal } \\
\text { shadow mask }\end{array}$ & Glass & 153 \\
\hline $\mathrm{MoS}_{2}$ & PSA & PSA antibody & $375 \mathrm{fM}$ & $\begin{array}{l}\text { Mechanical exfoliation, photoli- } \\
\text { thography, thermal evaporation }\end{array}$ & Silicon & 154 \\
\hline $\mathrm{MoS}_{2}$ & PSA & PSA antibody & $1 \mathrm{pg} / \mathrm{ml}$ & $\begin{array}{l}\text { Mechanical exfoliation, photoli- } \\
\text { thography, thermal evaporation }\end{array}$ & Silicon & 155 \\
\hline $\mathrm{MoS}_{2}$ & TNF- $\alpha$ & TNF- $\alpha$ antigen & $60 \mathrm{fM}$ & $\begin{array}{l}\text { Exfoliation, photolithography, } \\
\text { lift-off process, atomic layer } \\
\text { deposition (ALD) }\end{array}$ & Silicon & 157 \\
\hline $\begin{array}{l}\text { Pt NP } \\
\text { decorated } \\
\mathrm{MoS}_{2}\end{array}$ & BNP & BNP-antibody & $100 \mathrm{fM}$ & $\begin{array}{l}\text { Photolithography, e-beam evapo- } \\
\text { ration }\end{array}$ & Silicon & 158 \\
\hline
\end{tabular}
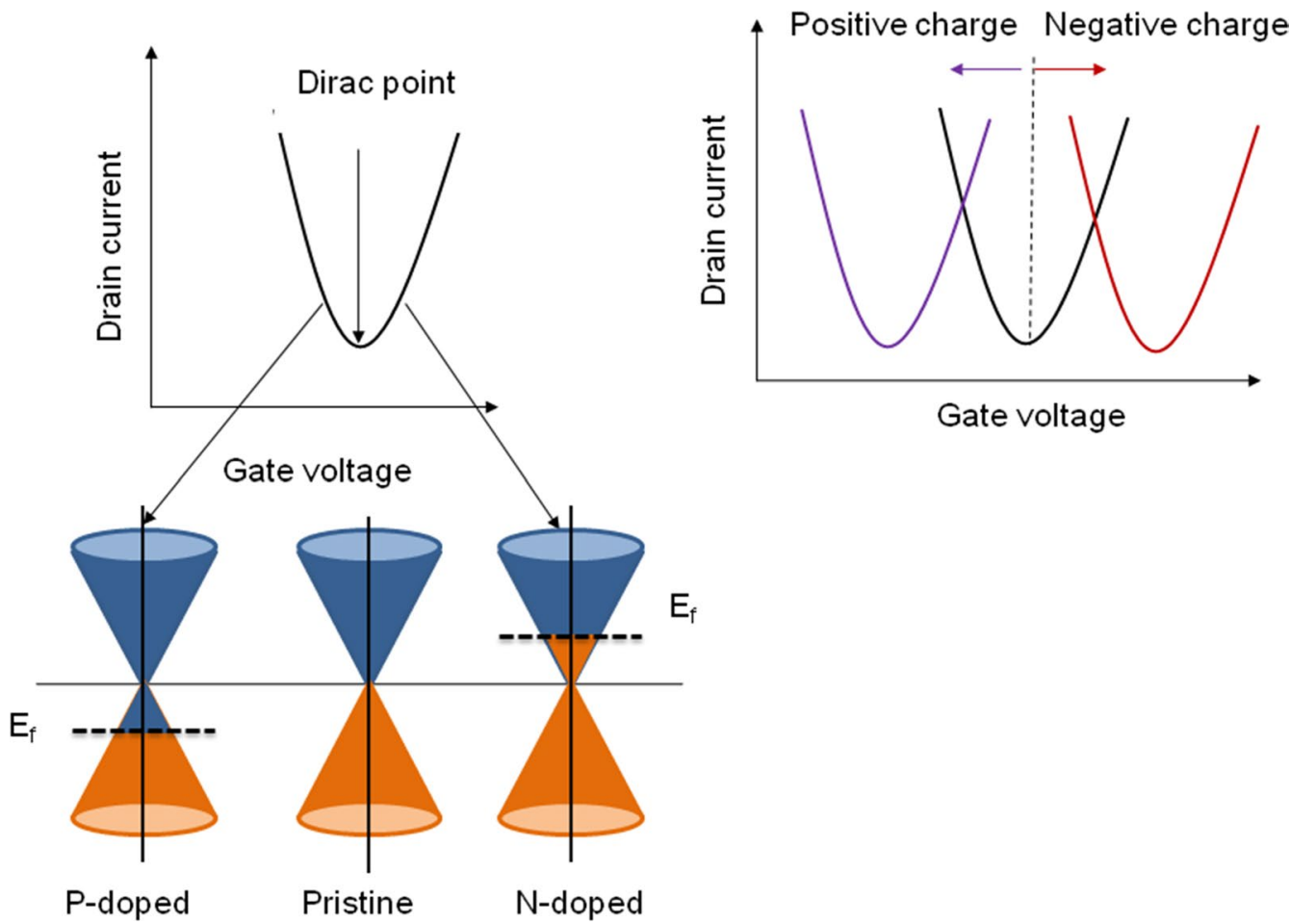

Fig. 14 Band diagram of graphene. The shift in Dirac point which governs the conductivity and hence the sensing mechanism has been shown here. 
of DNA with an LOD of $\sim 25 \mathrm{nM}$. The dynamic range of the device encompassed over five orders of concentration magnitude with excellent selectivity to detect single-base mismatches. Stine et al. ${ }^{142}$ fabricated a label-free DNA detection biosensor based on a reduced graphene oxide (rGO) FET which could detect 2 nM DNA. Cai et al. ${ }^{143}$ fabricated an rGO-based FET biosensor for DNA sensing in which peptide nucleic acid (PNA) was employed as the capture probe. The hybridization of integral DNA with the probe PNA caused $n$ doping of the sensor that led to a shift of the Dirac point. In this study, the reusability of the sensors was explored by immersing the sensors in a urea solution. The sensor signal stayed at $96.67 \%$ and $83.33 \%$ in the second and the third cycles, respectively, demonstrating the robustness of the device when subjected to unintentional liquid exposure. The surface defects of rGO make the immobilization procedure much easier, in which the involved radicals are hydroxyl, carbonyl, or oxygen groups. While the defect sites are not uniform and difficult to control precisely, CVD grown graphene has provided advantages in its uniform binding and controllability via surface modification of the graphene structure by utilizing $\pi-\pi$ interactions.

Zheng and co-workers ${ }^{144}$ developed CVD-grown singlelayer graphene that was transferred onto a pre-fabricated sensor array surface (directional transfer technique) for highly specific detection of DNA having a detection limit of 10 fM with good reproducibility and stability. This directional transfer technique has advantages such as simple operation and a higher yield of usable graphene structures compared to conventional transfer techniques. Cai et al. ${ }^{145}$ reported a $\mathrm{Au}$ NP-decorated G-FET biosensor to analyse miRNA with a detection limit of $10 \mathrm{fM}$.

Lee's group ${ }^{146}$ utilized $\mathrm{MoS}_{2}$ nanosheets as a channel material to analyse DNA hybridization, fabricated through the reaction of $\mathrm{H}_{2} \mathrm{~S}$ gas with a patterned Mo layer. The metal electrodes were passivated by SU-8 epoxy resin to reduce leakage current and biomolecules adsorption, as shown in Fig. 15. This $\mathrm{MoS}_{2}$-based FET sensing device could detect down to $10 \mathrm{fM}$ of DNA with a high sensitivity of $17 \mathrm{mV} /$ decade and had a high dynamic range of $10^{6}$.

As can be seen from the earlier studies, 2D nanomaterials provide extremely low detection limits, enable label-free detection of target nucleic acids without any pre-amplification, giving real-time, continuous quantification of nucleic acids as opposed to PCR, which is not compatible with continuous monitoring of biological samples. Moreover, device fabrication of such nano Bio-FETs is generally compatible with standard lithographic techniques, advantageous for mass production. Park et al. ${ }^{147}$ investigated the effect of doxorubicin on DNA nanostructures with a copper ion/ $\mathrm{MoS}_{2}$ hybrid structure in which $\mathrm{Cu}^{2+}$ induced a positive charge in the DNA receptor. This positive charge of the $\mathrm{Cu}^{2+}$ could enable the sensing ability of negatively charged doxorubicin (target). The high sensitivity of $1.7 \times 10^{3} \mathrm{~A} / \mathrm{A}$ was achieved

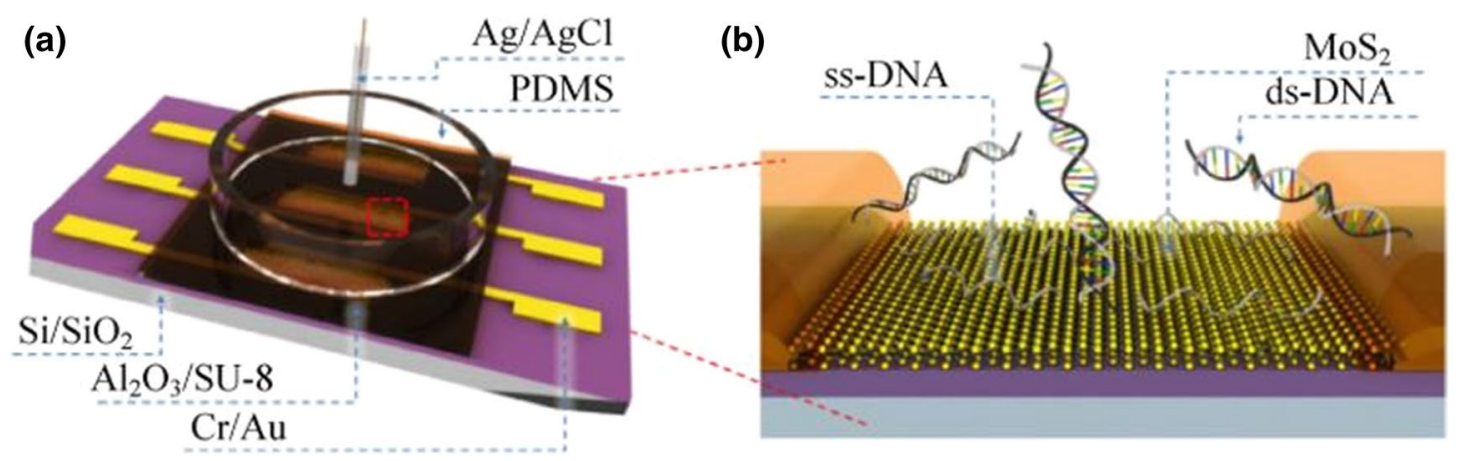

(c)

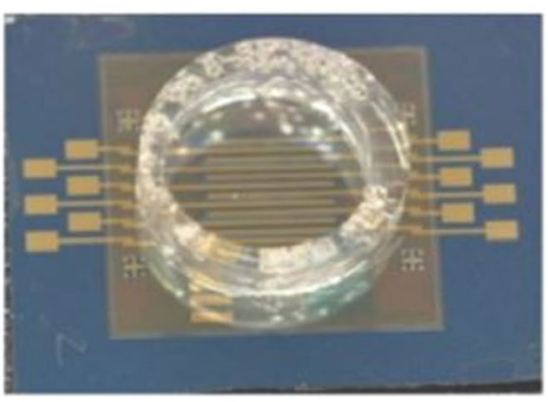

(d)

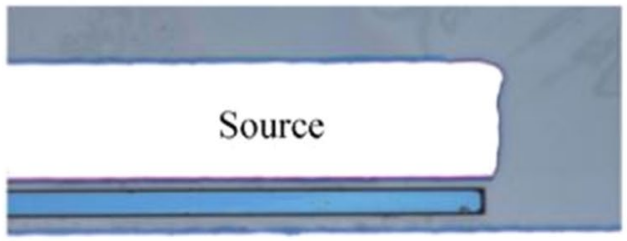

Drain
Fig. 15 (a) Schematic illustration of a $\mathrm{MoS}_{2}$ device operated by solution gating for DNA detection. (b) Configuration of a $\mathrm{MoS}_{2}$ Bio-FET. (c) and (d) are optical images of multiple and single $\mathrm{MoS}_{2}$ devices, respectively. Reprinted from Yan et al. ${ }^{148}$ under the terms of Creative Commons (CC BY 3.0) license. 
in this $\mathrm{Cu}^{2++}$-DNA/MoS ${ }_{2}$-based Bio-FET which is due to the minimal distance between the biomolecules and sensor surface and without the usage of a high-k dielectric layer between the channel region and the receptor. From this result, it was concluded that the sensitivity of the Bio-FET mainly relies on the dielectric constant and thickness of the dielectric material.

Recently, Majid et al. ${ }^{149}$ proposed a label-free biosensor using $\mathrm{MoS}_{2}$ for ultrasensitive detection of miRNA155 , a breast cancer biomarker in human serum. The probe miRNA-155 was immobilized onto $\mathrm{MoS}_{2}$ by physical adsorption. This device showed a low subthreshold swing of $48.10 \mathrm{mV} /$ decade and a low LOD of $0.03 \mathrm{fM}$ with a linear range from $0.1 \mathrm{fM}$ to $10 \mathrm{nM}$. Figure 16 shows the evolution in employing nanomaterials for DNA detection.

\section{Bio-FETs for DNA Detection: A Summary}

Bio-FETs are an alternate approach to the common methods for the detection of DNA without the requirement of labelling. G-FET- and TMDC-based Bio-FETs are the most common designs that have been extensively employed in this regard, owing to high sensitivity, high transparency, and ease of manufacturing and integration into on-chip device fabrication. CVD-grown single-layer graphene has shown the most promise for detecting DNA down to $100 \mathrm{fM}$, but the most significant challenge is in preparing and transferring into the device architecture of high-quality graphene without surface contamination. Moreover, the small on/off current ratio, due to the lack of a bandgap in graphene, hinders their practical utility and inhibits the sensitivity of the device due to a high possibility of interfering species causing the sensing response. TMDC materials are also an attractive material class due to ease and maturity of fabrication processes, lower cost (compared to mono-layer graphene synthesis), and similar sensing performance, thereby showing immense potential for realizing DNA sensors. This has been expounded by a study reviewed here which has demonstrated that in a direct comparison with a GFET-based DNA sensor, a $\mathrm{MoS}_{2}$-based Bio-FET device could sense down to $10 \mathrm{fM}$ DNA with a high sensitivity of $17 \mathrm{mV} / \mathrm{dec}$. Further improvements in TMDC devices, specifically in terms of improving the sensing properties such as response time and repeatability, can further TMDCs for use in DNA detection.

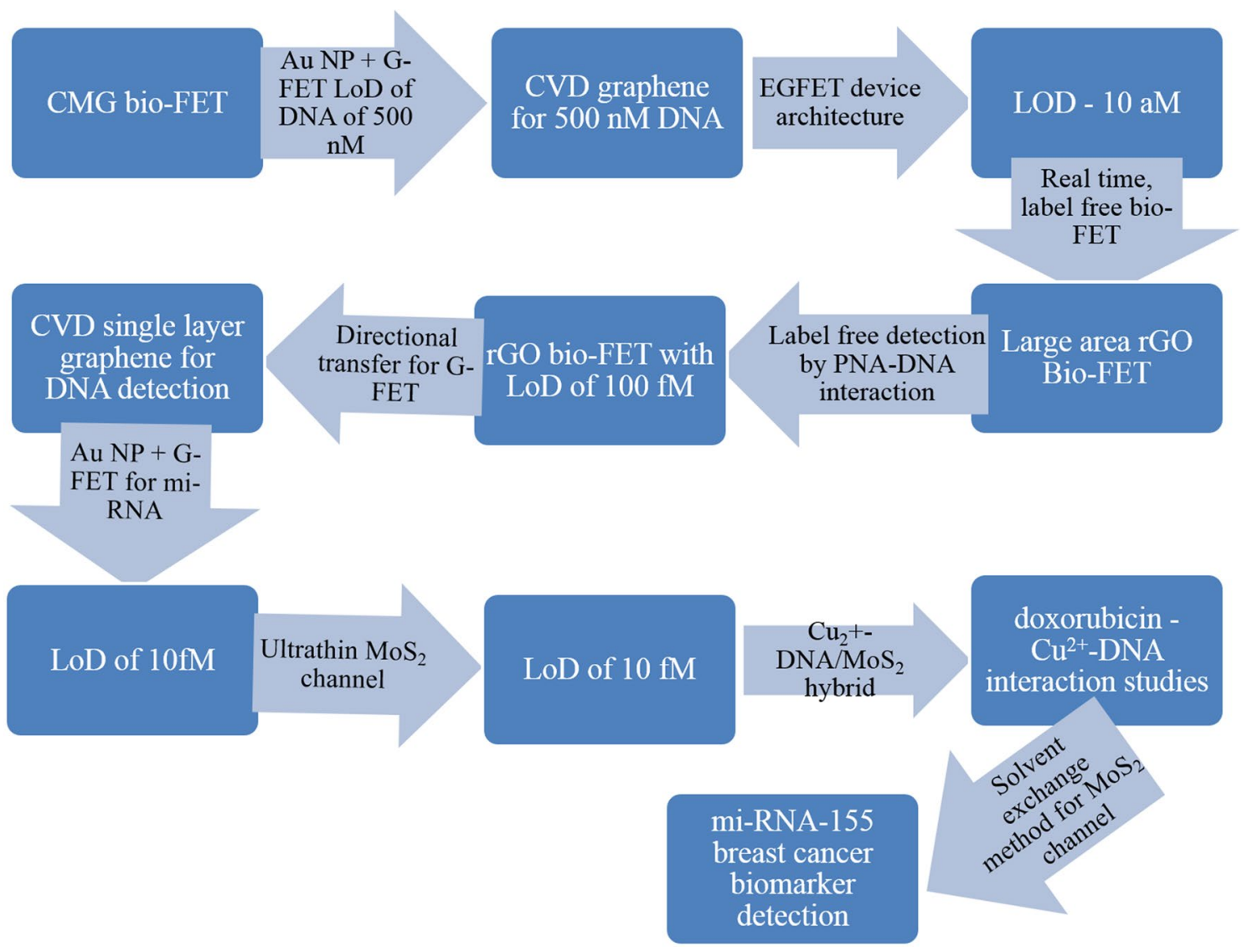

Fig. 16 Timeline of DNA detection through nanomaterial-incorporated FET sensors. 


\section{Bio-FETs for Other Analytes}

Immunosensors are the class of biosensors that facilitate quantifying and monitoring the functioning of the immune system; they can specifically detect the antibodies or antigens through the antigen-antibody interactions in biological samples. Apart from the most common analytes detailed above, immunosensors have wider applications in the early detection of disease biomarkers, environmental monitoring, and even food safety. The analyte is a microorganism such as a virus or bacterium. Although the immunosensors have been utilized in a variety of transducing mechanisms, this review is focused on immunosensors based on FET. Immunosensors based on Bio-FET sensing platform offer several advantages such as miniaturization, integration into an electronic chip, reusability, and use in portable devices.

\section{Detection of Proteins}

Ohno et al. ${ }^{150}$ examined single-layer graphene-based FET biosensors for bovine serum albumin (BSA) detection. They have also investigated an aptamer-modified graphene Bio-FET for selective detection of immunoglobulin ( $\operatorname{IgE}$ ) for specific detection of IgE protein. Sarkar et al. ${ }^{59}$ demonstrated a $\mathrm{MoS}_{2}$-based biosensor with a $\mathrm{HfO}_{2}$ gate dielectric for detection of biotin-streptavidin interactions at a concentration of $100 \mathrm{fM} . \mathrm{MoS}_{2}$ has free dangling bonds which reduce the surface roughness scattering and interface traps. It leads to better electrostatic control and reduces the noise of the device. Due to the hydrophilic nature of dielectrics like $\mathrm{HfO}_{2}$, it has a low affinity to biomolecule adsorption. Although this issue can be circumvented by additional surface functionalization using molecules such as APTES, it affects device sensitivity due to the ionic screening of such functionalizing molecules. The material combination in this study was investigated for $\mathrm{pH}$ detection and had sensitivity 74 times higher compared to graphene-based biosensors. In Bio-FETs, $n$-type or $p$-type semiconducting material with a bandgap act as a channel in which the electrons possessing higher energy than the source-to-channel barrier will begin to flow from source to drain. When the semiconducting material bandgap is extremely small, more electrons are mobilized to the drain from the source. Due to the zero bandgap of graphene and extreme thinness of the barrier, the electrons are transported from the source to drain through tunnelling. This promotes an increase in leakage current in off-state conditions and leads to low sensitivity.

\section{Detection of viruses}

Seo et al. ${ }^{151}$ developed a graphene-based biosensing device for the detection of SARS-CoV-2 virus (COVID-19) in which SARS-CoV-2 antigen was functionalized with the graphene channel material. The performance of the sensor was demonstrated using antigen protein, cultured virus, and nasopharyngeal swab specimens from COVID-19 patients. They measured the transfer curves of the G-FET after pyrenebutanoic acid succinimidyl ester (PBASE) functionalization which showed a positive shift due to the $p$ doping effect of the pyrene group. The transfer curve was shifted negatively, suggesting that the positive charge of the antibody exerted an $n$-doping effect on graphene. This FET sensor could detect concentrations of $1 \mathrm{fg} / \mathrm{ml}$ in PBS and $100 \mathrm{fg} / \mathrm{ml}$ of clinical transport medium (for preserving viral viability). Moreover, enzyme-linked immunoassay (ELISA) confirmed that the antibody is selectively bound to the SARS-CoV-2 antigen and that it does not interfere with MERS-CoV spike protein and BSA. ${ }^{151}$ Figure 17 presents a picture of proteins, cancer biomarkers, and viruses that have been studied with nano Bio-FETs.

\section{Detection of Cancer Biomarkers}

Myng et al. ${ }^{152}$ studied the detection of cancer biomarkers with an r-GO-encapsulated nanoparticle biosensor with a detection limit of $1 \mathrm{pM}$ for human epidermal growth factor receptor-2 (HER2) and $100 \mathrm{pM}$ for epidermal growth factor receptor (EGFR) target cancer biomarkers. Kim et al. ${ }^{153}$ used $\mathrm{r}-\mathrm{GO}$ for ultrasensitive detection of prostate-specific antigen (PSA) as a cancer biomarker. To immobilize the PSA monoclonal antibody $(\mathrm{m}-\mathrm{Ab})$ on the $\mathrm{r}-\mathrm{GO}$ channel, the channel layer was first covered with an $\mathrm{Al}_{2} \mathrm{O}_{3}$ dielectric + PDDA layer followed by surface treatment with PBASE linker post which PSA m-Ab was added. The interaction between antigen and antibody was proportional to the analyte protein concentration with a wide dynamic range of up to $10^{6}$ in 1 $\mu \mathrm{M}$ PBS solution and an LOD as low as $100 \mathrm{fg} / \mathrm{ml}$.

A bio-functionalized $\mathrm{MoS}_{2}$ nanosheet FET device for cancer marker detection was demonstrated by Wang et al. ${ }^{154}$ An $\mathrm{HfO}_{2}$ dielectric deposited on a mechanically exfoliated $\mathrm{MoS}_{2}$ surface in a solution-gated FET configuration was employed for PSA detection. The channel conductance of the $n$-type FET increased when negatively charged PSA was bound to the antibody receptor. Sensitivity to concentrations up to $375 \mathrm{fM}$ with good specificity and rapid detection was reported. In addition to functioning as a gate dielectric, $\mathrm{HfO}_{2}$ also reduces the loss of binding sites due to the hydrophilic nature and protects metal electrodes from ionic degradation, thereby enhancing the life of the device.

An oxide-free approach of $\mathrm{MoS}_{2}$ Bio-FET fabrication has been explored to detect PSA by Lee et al. ${ }^{155} \mathrm{MoS}_{2}$ 


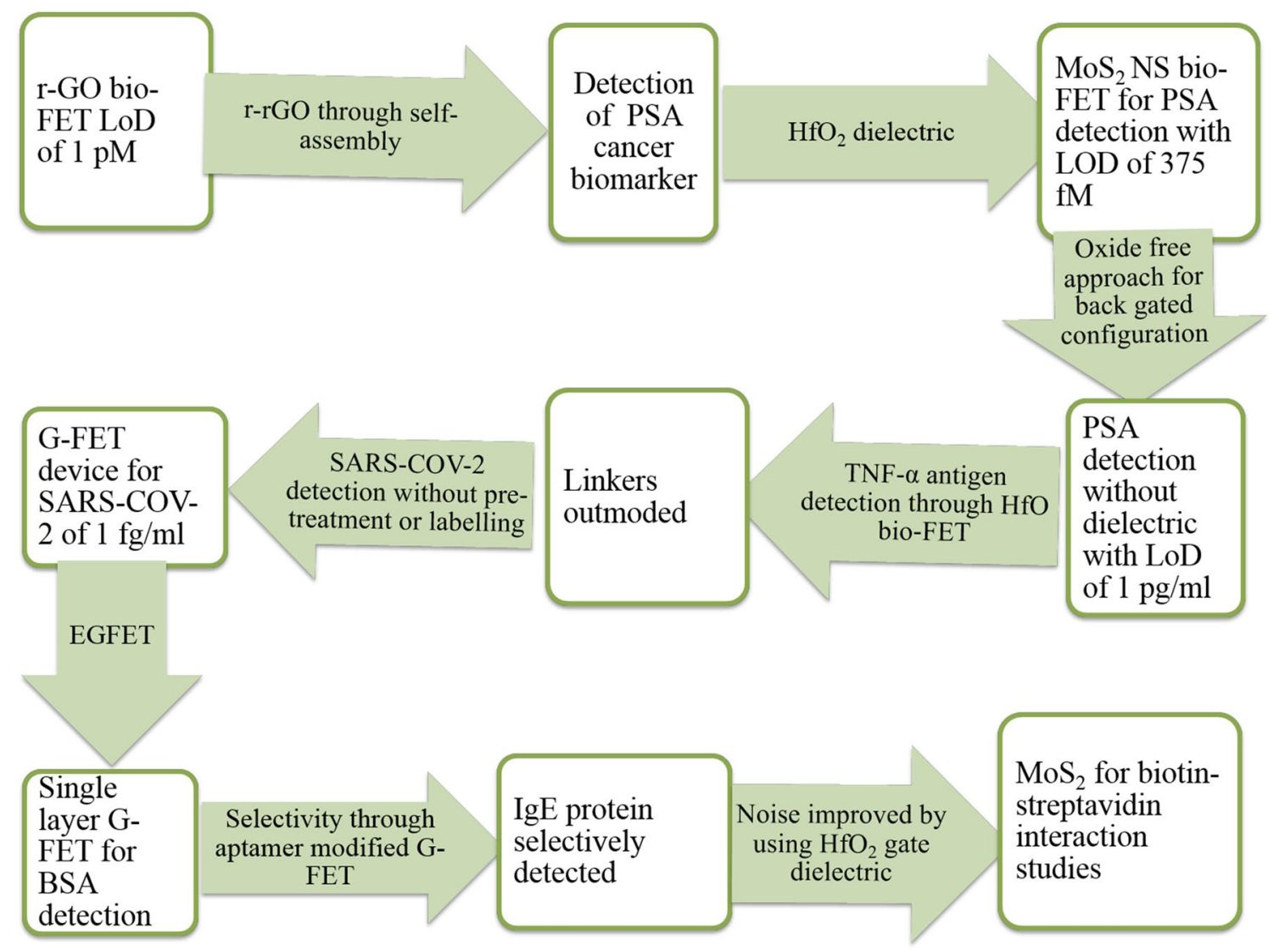

Fig. 17 Timeline of investigation of nanomaterials for the detection of proteins, viruses, and cancer biomarkers.

nanosheets had a $75.77^{\circ}$ contact angle with water, confirming its hydrophobic nature which suits biomolecular interactions. The hydrophobicity of $\mathrm{MoS}_{2}$ has a higher affinity for biomolecular-surface adsorption than the hydrophilic surfaces. ${ }^{156}$ The specific antigen was detected by the back-gated $\mathrm{MoS}_{2}$ Bio-FET without the need for a dielectric layer on the top of the channel, simplifying and thereby strengthening the device design. This $\mathrm{MoS}_{2}$ Bio-FET sensor could achieve an LOD of up to $1 \mathrm{pg} / \mathrm{ml}$ which is considerably lower than the clinical cutoff level of $-4 \mathrm{ng} / \mathrm{ml}$.

Nam et al. ${ }^{157}$ investigated the differences between a HfOcoated and HfO-free $\mathrm{MoS}_{2}$ Bio-FET sensor for tumour necrosis factor (TNF- $\alpha$ ) antigen detection. For the HfO layer-covered sensor, antibody receptors were functionalized on the HfO layer. The charge exchange with the TNF- $\alpha$ target molecule through antigen-antibody binding was only capacitively coupled with the $\mathrm{MoS}_{2}$ channel and moved the threshold voltage without changing its mobility or transconductance. For the HfO layer-free sensor, antibodies were directly bound on the $\mathrm{MoS}_{2}$ channel, which modulated its $\mathrm{ON}$-state transconductance. This was due to the disordered potential formation in the $\mathrm{MoS}_{2}$ channel which contributed to the reduction of FET mobility. Moreover, HfO layer-free sensors could enable the easy surface functionalization processes without using complicated linkers. Lei et al. ${ }^{158}$ introduced a label-free biosensor based on Pt NPs added to rGO for the detection of brain natriuretic peptide (BNP) in blood wherein the LOD was down to $100 \mathrm{fM}$.

\section{Bio-FETs for Cancer Biomarker Detection: A Summary}

The sensitivity of a Bio-FET highly relies on the thickness of the dielectric, its dielectric constant, and the interface between the channel and the dielectric. For instance, if the thickness of the dielectric is less, the transconductance will increase consequently and lower the operating voltage. However, after a minimum thickness, this also leads to a leakage current, rendering the device useless for detection.

In general, it is seen that $\mathrm{MoS}_{2}$ Bio-FETs for cancer marker detection can have high sensitivity, high flexibility, and transparency, especially when compared to SiNW, CNT, and G-FET biosensors. ${ }^{46}$ The pristine $\mathrm{MoS}_{2}$ surface is free of dangling bonds, which reduces the low density of electron scattering centres and surface roughness. This in turn leads to lower electronic noise, one of the main sources of noise in FET biosensors. The direct energy bandgap of $\mathrm{MoS}_{2}$ also significantly reduces the leakage current compared to G-FET 
sensors for example. ${ }^{159}$ However, $\mathrm{MoS}_{2}$ Bio-FETs still suffer from instability and poor reusability due to decomposition from side reactions during detection, such as those between the reaction products and the electrodes. To overcome this, $\mathrm{MoS}_{2}$ with other polymer materials such as PANI, poly (diallyl dimethylammonium chloride) (PDDA), and polydopamine (PDA) for electrode passivation may be used as channel materials for biosensing applications, with improved stability and sensitivity.

\section{Summary and Challenges}

In this review article, we have highlighted the potential of the Bio-FET device architecture for detecting multiple analytes since their use with nanomaterials. Nanomaterial-based Bio-FET sensing over the last decade has employed novel materials that are continuously evolving with new morphologies, chemistries, and architectures to improve sensing performance. Since the advent and requirement of flexible sensors with low power consumption, Bio-FETs, in general, have garnered their importance in the sensing field due to multiple prospects such as repeatable fabrication and device performance, and optimized costs considering improvements to device yield and portability for use in POCT for example. With such progress, implantable devices are indispensable as the next evolutionary step and indeed can be proposed to be inevitable considering the pace of progress in the relevant fields of research for a wide variety of applications. The major challenges in nanomaterial Bio-FETs that remain to be solved, in the opinion of the authors, are important for formulating solutions for addressing these challenges.

Two-dimensional nanomaterials such as graphene and $\mathrm{MoS}_{2}$ offer unique electronic properties, high surface area, high carrier mobility, and a tunable bandgap, making them a promising emerging sensing platform for Bio-FET-based biological detection.

Compared with 1D nanomaterials such as CNTs and other NWs such as SiNW, and even G-FET biosensors, $\mathrm{MoS}_{2}$-based FET biosensors exhibit high sensitivity, high flexibility, transparency, device scalability, easy device fabrication, and large-scale integrability in the lab scale. An evolutionary 2D nanomaterial seems to be $\mathrm{WSe}_{2}$, which, when employed for Bio-FET sensors, exhibits a higher ON state owing to its ambipolar transport property in comparison with $\mathrm{MoS}_{2}$ sensors. These 2D nanomaterial Bio-FETs face a predominant challenge in terms of high-throughput device fabrication. Specifically, there is still no repeatable process for multiple material classes for precise placement/ transfer of the device architecture onto a synthesized 2D nanomaterial or vice versa. This is a primary reason why the greater part of 2D Bio-FETs is only at the lab scale. Developing the scalability in terms of the transfer techniques will revolutionize the field penetration of Bio-FETs. Another technicality involving 2D materials is achieving efficient regeneration of the molecular recognition layer, which has direct implications in the cost. Although costs can be reduced by multiple directions such as switching to alternative materials and by device structure optimizations, a simpler approach can be by improving the reusability of the sensor by achieving efficient regeneration of the sensing layer or improving the stability of the electrodes and contacts.

Bio-FETs need to be biocompatible (for achieving implantable devices) and environmentally stable to increase robustness, for example in the case of wearable devices, and to increase shelf life. In the case of implantable devices, the sensor chip will necessarily come in contact with complex biological environments, which can degrade the device performance quickly. To address this issue, systematic investigations of 2D material stability such as coating and storage strategies are being incessantly developed. One approach to improve stability/reusability is to have the sensing area separated from the electrode area wherein connections can be made between the two sections.

Another challenge to achieving better commercialization is to understand the specifics of the interaction mechanisms between biological molecules and the sensing materials. A few of the phenomena, such as anomalous differences in sensitivity to biomolecules based on differences and nature of binding to the sensing layer, kinetics of adsorption and desorption in the presence of interfering molecules, the influence of contaminants and the tendency for their repetitive presence during different stages of material/device fabrication, and the dynamics of charge transfer between enzymes and the sensing layer, need further appreciation in this regard. It can be envisioned hence that with the advent of promising technologies in multiple fronts, nanomaterialbased Bio-FETs have huge potential for commercial biomedical devices.

Acknowledgments The authors wish to acknowledge and thank the facilities and fellowship provided by the Management, PSG Sons \& Charities, Coimbatore, India

Conflict of interest The authors declare no conflict of interest.

\section{References}

1. T. Wadhera, D. Kakkar, G. Wadhwa, and B. Raj, J. Electron. Mater. 48, 7635 (2019).

2. B.D. Malhotra, and C.M. Pandey, Book-Biosensors : Fundamentals and Applications (2017).

3. J. Go, P.R. Nair, and M.A. Alam, J. Appl. Phys. 112, 034516 (2012).

4. A. Sassolas, L.J. Blum, and B.D. Leca-Bouvier, Biotechnol. Adv. 30, 489 (2012).

5. L.C. Clark, and C. Lyons, Ann. N. Y. Acad. Sci. 102, 29 (1962). 
6. A. Aziz, M. Asif, G. Ashraf, M. Azeem, I. Majeed, M. Ajmal, J. Wang, and H. Liu, Microchim. Acta 186, 1 (2019).

7. C. Liu, C. Xu, N. Xue, J.H. Sun, H. Cai, T. Li, Y. Liu, and J. Wang, MEMS Sensors - Des. Appl. 49, 49 (2018).

8. A. Nehra, and K. Pal Singh, Biosens. Bioelectron. 74, 731 (2015).

9. L. Manjakkal, D. Szwagierczak, and R. Dahiya, Prog. Mater. Sci. 109, 100635 (2020).

10. G.C. Mak, P.K. Cheng, S.S. Lau, K.K. Wong, C.S. Lau, E.T. Lam, R.C. Chan, and D.N. Tsang, J. Clin. Virol. 129, 104500 (2020).

11. M.I. Mohammed, and M.P.Y. Desmulliez, Lab Chip 11, 569 (2011).

12. P. Salvo, V. Dini, A. Kirchhain, A. Janowska, T. Oranges, A. Chiricozzi, T. Lomonaco, F. Di Francesco, and M. Romanelli, Sensors 17, 10 (2017).

13. A. Syahir, K. Usui, K. Tomizaki, K. Kajikawa, and H. Mihara, Microarrays 4, 228 (2015).

14. W.V. Gonzales, A.T. Mobashsher, and A. Abbosh, The Progress of Glucose Monitoring - A Review of Invasive to Minimally and Non-Invasive Techniques, Devices and Sensors (2019).

15. E. Stern, A. Vacic, N.K. Rajan, J.M. Criscione, J. Park, B.R. Ilic, D.J. Mooney, M.A. Reed, and T.M. Fahmy, Nat. Nanotechnol. 5, 138 (2010).

16. P. C. Chen, Y. W. Chen, I. Sarangadharan, C. P. Hsu, C. C. Chen, S. C. Shiesh, G. Bin Lee, and Y. L. Wang, ECS J Solid State Sci. Technol. 6, Q71 (2017).

17. N. Tripathy, and D.H. Kim, Nano Converg. 5, 1 (2018).

18. R. Ahmad, T. Mahmoudi, M.S. Ahn, and Y.B. Hahn, Biosens. Bioelectron. 100, 312 (2018).

19. Y.-C. Syu, W.-E. Hsu, and C.-T. Lin, ECS J. Solid State Sci. Technol. 7, Q3196 (2018).

20. D. Wang, V. Noël, and B. Piro, Electronics 5, 9 (2016).

21. S.K. Arya, S. Saha, J.E. Ramirez-Vick, V. Gupta, S. Bhansali, and S.P. Singh, Anal. Chim. Acta 737, 1 (2012).

22. K. Ogata, K. Koike, T. Tanite, T. Komuro, S. Sasa, M. Inoue, and M. Yano, Sensors Actuators B Chem. 100, 209 (2004).

23. T.H. Yang, T.Y. Chen, N.T. Wu, Y.T. Chen, and J.J. Huang, IEEE Trans. Electron Devices 64, 1286 (2017).

24. B. Kannan, D.E. Williams, C. Laslau, and J. Travas-sejdic, Biosens. Bioelectron. 35, 258 (2012).

25. D. Elkington, N. Cooling, W. Belcher, P.C. Dastoor, and X. Zhou, Electronics 3, 234 (2014).

26. S.J. Park, H.S. Song, O.S. Kwon, J.H. Chung, S.H. Lee, J.H. An, S.R. Ahn, J.E. Lee, H. Yoon, T.H. Park, and J. Jang, Sci. Rep. 4, 1 (2014).

27. S.J. Park, S.H. Lee, H. Yang, C.S. Park, C.S. Lee, O.S. Kwon, T.H. Park, and J. Jang, ACS Appl. Mater. Interfaces 8, 28897 (2016).

28. J. Liu, M. Agarwal, and K. Varahramyan, Sensors Actuators $B$ Chem. 135, 195 (2008).

29. R. Elnathan, M. Kwiat, A. Pevzner, Y. Engel, L. Burstein, A. Khatchtourints, A. Lichtenstein, R. Kantaev, and F. Patolsky, Nano Lett. 12, 5245 (2012).

30. G. Zheng, X.P.A. Gao, and C.M. Lieber, Nano Lett. 10, 3179 (2010).

31. W. Chen, H. Yao, C.H. Tzang, J. Zhu, M. Yang, and S.T. Lee, Appl. Phys. Lett. 88, 5 (2006).

32. E. Stern, R. Wagner, F.J. Sigworth, R. Breaker, T.M. Fahmy, and M.A. Reed, Nano Lett. 7, 3405 (2007).

33. D. Lee, and T. Cui, Biosens. Bioelectron. 25, 2259 (2010).

34. G.S. Kulkarni, and Z. Zhong, Nano Lett. 12, 719 (2012).

35. C. Andronescu, and W. Schuhmann, Curr. Opin. Electrochem. 3, 11 (2017).

36. Y. Hu, Y. Huang, C. Tan, X. Zhang, Q. Lu, M. Sindoro, X. Huang, W. Huang, L. Wang, and H. Zhang, Mater. Chem. Front. 1, 24 (2017).
37. S. Wu, Z. Zeng, Q. He, Z. Wang, S.J. Wang, Y. Du, Z. Yin, X. Sun, W. Chen, and H. Zhang, Small 8, 2264 (2012).

38. A. Kumar, N. Gupta, M. M. Tripathi, and R. Chaujar, Microsyst. Technol. 6, (2019).

39. Y. Chen, R. Ren, H. Pu, J. Chang, S. Mao, and J. Chen, Biosens. Bioelectron. 89, 505 (2017).

40. A. Pon, A. Bhattacharyya, and R. Rathinam, J. Electron. Mater. 50, 6020 (2021).

41. S. Rollo, D. Rani, W. Olthuis, and C. Pascual García, Biophys. Rev. 11, 757 (2019).

42. S. Sang, Y. Wang, Q. Feng, Y. Wei, J. Ji, and W. Zhang, Crit. Rev. Biotechnol. 36, 465 (2016).

43. H. Tang, P. Lin, H.L.W. Chan, and F. Yan, Biosens. Bioelectron. 26, 4559 (2011).

44. M. Zhang, C. Liao, Y. Yao, Z. Liu, F. Gong, and F. Yan, Adv. Funct. Mater. 24, 978 (2014).

45. A.C.M. de Moraes, and L.T. Kubota, Chemosensors 4, 20 (2016).

46. S. Su, Q. Sun, X. Gu, Y. Xu, J. Shen, D. Zhu, J. Chao, C. Fan, and L. Wang, Trends Anal. Chem. 119, 115610 (2019).

47. W. Choi, N. Choudhary, G.H. Han, J. Park, D. Akinwande, and Y.H. Lee, Mater. Today 20, 116 (2017).

48. H. Hu, A. Zavabeti, H. Quan, W. Zhu, H. Wei, D. Chen, and J.Z. Ou, Biosens. Bioelectron. 142, 111573 (2019).

49. M. Kaisti, Biosens. Bioelectron. 98, 437 (2017).

50. V. Naresh, and N. Lee, Sensors 21, 1 (2021).

51. P. Lin, and F. Yan, Adv. Mater. 24, 34 (2012).

52. T. Kuila, S. Bose, P. Khanra, A.K. Mishra, N.H. Kim, and J.H. Lee, Biosens. Bioelectron. 26, 4637 (2011).

53. N. Bhalla, P. Jolly, N. Formisano, and P. Estrela, Essays Biochem. 60, 1 (2016).

54. D. Grieshaber, R. MacKenzie, J. Vörös, and E. Reimhult, Sensors $8,1400(2008)$

55. K. Jenifer, S. Arulkumar, S. Parthiban, and J.Y. Kwon, J. Electron. Mater. 49, 7098 (2020).

56. J. Xu, X. Luo, and H. Chen, Anal. Chem. 10, 420 (2005).

57. S. Carrara and K. Iniewski, Handbook of Bioelectronics: Directly Interfacing Electronics and Biological Systems (2015).

58. A. Tixier-Mita, S. Ihida, B. D. Ségard, G. A. Cathcart, T. Takahashi, H. Fujita, and H. Toshiyoshi, Jpn. J. Appl. Phys. 55, 04EA08 (2016).

59. D. Sarkar, W. Liu, X. Xie, A.C. Anselmo, S. Mitragotri, and K. Banerjee, ACS Nano 8, 3992 (2014).

60. L.G. Chen, and M.S.C. Lu, Micro Nano Lett. 6, 482 (2011).

61. X. Chen, C. Liu, and S. Mao, Nano-Micro Lett. 12, 1 (2020).

62. C.A. Vu, and W.Y. Chen, Sensors 19, 4214 (2019).

63. L. Torsi, M. Magliulo, K. Manoli, and G. Palazzo, Chem. Soc. Rev. 42, 8612 (2013).

64. L. Syedmoradi, A. Ahmadi, M.L. Norton, and K. Omidfar, Microchim. Acta 186, 1 (2019).

65. S.H. Lee, J.H. Sung, and T.H. Park, Ann. Biomed. Eng. 40, 1384 (2012).

66. T.K. Aparna, and R. Sivasubramanian, J. Nanosci. Nanotechnol. 18, 789 (2018)

67. P.R. Solanki, A. Kaushik, V.V. Agrawal, and B.D. Malhotra, NPG Asia Mater. 3, 17 (2011).

68. S. Kumar, S. D. Bukkitgar, S. Singh, Pratibha, V. Singh, K. R. Reddy, N. P. Shetti, C. Venkata Reddy, V. Sadhu, and S. Naveen, ChemistrySelect 4, 5322 (2019).

69. K. Kalantar-Zadeh, and J.Z. Ou, ACS Sensors 1, 5 (2016).

70. A. Liu, Biosens. Bioelectron. 24, 167 (2008).

71. N. Mohanty, and V. Berry, Nano Lett. 8, 4469 (2008).

72. K. Movlaee, H. Raza, N. Pinna, S. G. Leonardi, and G. Neri, Electrochemical Glucose Sensor Based on NiO/CNT Composite (Springer International Publishing, n.d.).

73. S. Su, H. Sun, F. Xu, L. Yuwen, and L. Wang, Electroanalysis 25,2523 (2013). 
74. S.J. Park, H. Yang, S.H. Lee, H.S. Song, C.S. Park, J. Bae, O.S. Kwon, T.H. Park, and J. Jang, ACS Nano 11, 5950 (2017).

75. S.M. Siddeeg, Int. J. Electrochem. Sci. 15, 599 (2020).

76. T.K. Aparna, R. Sivasubramanian, and M.A. Dar, J. Alloys Compd. 741, 1130 (2018).

77. D.C. Li, P.H. Yang, and M.S.C. Lu, IEEE Trans. Electron Devices 57, 2761 (2010).

78. S. Casalini, F. Leonardi, T. Cramer, and F. Biscarini, Org. Electron. 14, 156 (2013).

79. T. Vijayaraghavan, R. Sivasubramanian, S. Hussain, and A. Ashok, ChemistrySelect 2, 5570 (2017).

80. T.K. Aparna, and R. Sivasubramanian, Mater. Today Proc. 5, 16111 (2018)

81. C. H. Lin, C. Y. Hsiao, C. H. Hung, Y. R. Lo, C. C. Lee, C. J. Su, H. C. Lin, F. H. Ko, T. Y. Huang, and Y. S. Yang, Chem. Commun. 5749 (2008).

82. B.R. Li, Y.J. Hsieh, Y.X. Chen, Y.T. Chung, C.Y. Pan, and Y.T. Chen, J. Am. Chem. Soc. 135, 16034 (2013).

83. T.T. Tran, and A. Mulchandani, TrAC - Trends Anal. Chem. 79, 222 (2016)

84. W. Yang, K.R. Ratinac, S.R. Ringer, P. Thordarson, J.J. Gooding, and F. Braet, Angew. Chemie - Int. Ed. 49, 2114 (2010).

85. G. Dharmalingam, R. Sivasubramaniam, and S. Parthiban, $J$. Electron. Mater. 49, 3009 (2020).

86. W.S. Li, P.X. Hou, C. Liu, D.M. Sun, J. Yuan, S.Y. Zhao, L.C. Yin, H. Cong, and H.M. Cheng, ACS Nano 7, 6831 (2013).

87. V. D. Bhatt, S. Joshi, K. Melzer, and P. Lugli, Proc. - 2016 IEEE Biomed. Circuits Syst. Conf. BioCAS 2016 1, 38 (2016).

88. S. Joshi, V.D. Bhatt, A. Märtl, M. Becherer, and P. Lugli, Biosensors 8, 1 (2018).

89. J. Park, S. J., Kwon, O. S., Lee, S. H., Song, H. S., Park, and T. H. Jang, Nano Lett. 12, 5082 (2012).

90. X. Huang, Z. Yin, S. Wu, X. Qi, Q. He, Q. Zhang, Q. Yan, F. Boey, and H. Zhang, Small 7, 1876 (2011).

91. J. Shim, H.Y. Park, D.H. Kang, J.O. Kim, S.H. Jo, Y. Park, and J.H. Park, Adv. Electron. Mater. 3, 1600364 (2017).

92. R. Sivasubramanian and P. Biji, Mater. Sci. Eng. B Solid-State Mater. Adv. Technol. 210, 10 (2016).

93. J. Fan, T. Li, and I. Djerdj, J. Electron. Mater. 44, 4080 (2015).

94. S. Rumyantsev, G. Liu, W. Stillman, V. Y. Kachorovskii, M. S. Shur, and A. A. Balandin, 21st Int. Conf. Noise Fluctuations, IEEE 234 (2011).

95. S.A. Pullano, C.D. Critello, I. Mahbub, N.T. Tasneem, S. Shamsir, S.K. Islam, M. Greco, and A.S. Fiorillo, Sensors 18, 4042 (2018).

96. N. Zagni, P. Pavan, and M. A. Alam, Appl. Phys. Lett. 114, (2019).

97. Z. Cheng, Q. Li, Z. Li, Q. Zhou, and Y. Fang, Nano Lett. 10, 1864 (2010).

98. F. Wang, L. Liu, and W.J. Li, IEEE Trans. Nanobioscience 14, 818 (2015).

99. Y. Kutovyi, I. Madrid, I. Zadorozhnyi, N. Boichuk, S.H. Kim, T. Fujii, L. Jalabert, A. Offenhaeusser, S. Vitusevich, and N. Clément, Sci. Rep. 10, 1 (2020).

100. R. Freeman, J. Elbaz, R. Gill, M. Zayats, and I. Willner, Chem. - A Eur. J. 13, 7288 (2007).

101. Y.S. Rim, J. Inf. Disp. 21, 203 (2020).

102. J. Kim, Y.S. Rim, H. Chen, H.H. Cao, N. Nakatsuka, H.L. Hinton, C. Zhao, A.M. Andrews, Y. Yang, and P.S. Weiss, ACS Nano 9, 4572 (2015).

103. N. Liu, L.Q. Zhu, P. Feng, C.J. Wan, Y.H. Liu, Y. Shi, and Q. Wan, Sci. Rep. 5, 1 (2015).

104. N. Liu, R. Chen, and Q. Wan, Sensors 19, 3425 (2019).

105. Y. Liu, B. Li, L. Zhu, P. Feng, Y. Shi, and Q. Wan, IEEE Electron Device Lett. 37, 778 (2016).

106. Z. Li, Y. Chen, Y. Xin, and Z. Zhang, Sci. Rep. 5, 1 (2015).
107. X. Luo, Z. Zhang, Q. Wan, K. Wu, and N. Yang, Electrochem. Commun. 61, 89 (2015)

108. N.S. Oliver, C. Toumazou, A.E.G. Cass, and D.G. Johnston, Diabet. Med. 26, 197 (2009).

109. J. Kim, A.S. Campbell, and J. Wang, Talanta 177, 163 (2018).

110. A. Heller, and B. Feldman, Chem. Rev. 108, 2482 (2008).

111. Z. Zhu, L. Garcia-Gancedo, A.J. Flewitt, H. Xie, F. Moussy, and W.I. Milne, Sensors 12, 5996 (2012).

112. K. Besteman, J.O. Lee, F.G.M. Wiertz, H.A. Heering, and C. Dekker, Nano Lett. 3, 727 (2003).

113. Y. Huang, X. Dong, Y. Shi, C.M. Li, L.J. Li, and P. Chen, Nanoscale 2, 1485 (2010).

114. Y.H. Kwak, D.S. Choi, Y.N. Kim, H. Kim, D.H. Yoon, S.S. Ahn, J.W. Yang, W.S. Yang, and S. Seo, Biosens. Bioelectron. 37, 82 (2012)

115. M. Zhang, C. Liao, C.H. Mak, P. You, C.L. Mak, and F. Yan, Sci. Rep. 5, 1 (2015).

116. K. Said, A.I. Ayesh, N.N. Qamhieh, F. Awwad, S.T. Mahmoud, and S. Hisaindee, J. Alloys Compd. 694, 1061 (2017).

117. N.A. Kumar, M.A. Dar, R. Gul, and J.B. Baek, Mater. Today 18, 286 (2015).

118. L. Gao, Small 13, 1 (2017).

119. S. Zeng, Z. Tang, C. Liu, and P. Zhou, Nano Res. 14, 1752 (2021).

120. K. Datta, A. Shadman, E. Rahman, and Q.D.M. Khosru, J. Electron. Mater. 46, 1248 (2017).

121. J. Ping, Z. Fan, M. Sindoro, Y. Ying, and H. Zhang, Adv. Funct. Mater. 27, 1605817 (2017).

122. H.W. Lee, D.H. Kang, J.H. Cho, S. Lee, D.H. Jun, and J.H. Park, ACS Appl. Mater. Interfaces 10, 17639 (2018).

123. J. Shan, J. Li, X. Chu, M. Xu, F. Jin, X. Wang, L. Ma, X. Fang, Z. Wei, and X. Wang, RSC Adv. 8, 7942 (2018).

124. N. Dalila R, M. K. Md Arshad, S. C. B. Gopinath, W. M. W. Norhaimi, and M. F. M. Fathil, Biosens. Bioelectron. 132, 248 (2019).

125. S. Maulik, S. Basu, K. Kanakamedala, and T. Daniels-Race, $J$. Electron. Mater. 48, 3451 (2019).

126. S. Barua, H.S. Dutta, S. Gogoi, R. Devi, R. Khan, ACS Appl Nano Mater. 1, 2 (2018).

127. S. M. Usman Ali, O. Nur, M. Willander, and B. Danielsson, IEEE Trans. Nanotechnol. 8, 678 (2009).

128. Y.B. Hahn, R. Ahmad, and N. Tripathy, Chem. Commun. 48, 10369 (2012).

129. M. Yano, K. Koike, K. I. Ogata, T. Nogami, S. Tanabe, and S. Sasa, Phys. Status Solidi Curr. Top. Solid State Phys. 9, 1570 (2012).

130. A. Janotti and C. G. Van De Walle, Reports Prog. Phys. 72, 126501 (2009)

131. H. Latif, S. Rasheed, A. Sattar, M.S. Rafique, S. Zaheer, A. Usman, M. Munam, and A. Imtiaz, J. Electron. Mater. 48, 7055 (2019).

132. M.S. Ahn, R. Ahmad, K.S. Bhat, J.Y. Yoo, T. Mahmoudi, and Y.B. Hahn, J. Colloid Interface Sci. 518, 277 (2018).

133. R. Ahmad, M.S. Ahn, and Y.B. Hahn, Electrochem. Commun. 77, 107 (2017).

134. M. Fathollahzadeh, M. Hosseini, M. Norouzi, A. Ebrahimi, M. Fathipour, M. Kolahdouz, and B. Haghighi, J. Solid State Electrochem. 22, 61 (2018).

135. X. Zong, and R. Zhu, Sensors Actuators B Chem. 255, 2448 (2018).

136. X. Du, Y. Li, J.R. Motley, W.F. Stickle, and G.S. Herman, ACS Appl. Mater. Interfaces 8, 7631 (2016).

137. I.K. Lee, K.H. Lee, S. Lee, and W.J. Cho, ACS Appl. Mater. Interfaces 6, 22680 (2014).

138. H. Chen, Y.S. Rim, I.C. Wang, C. Li, B. Zhu, M. Sun, M.S. Goorsky, X. He, and Y. Yang, ACS Nano 11, 4710 (2017). 
139. N.T. Nguyet, V. Van Thu, H. Lan, T. Trung, A.T. Le, V.H. Pham, and P.D. Tam, J. Electron. Mater. 48, 6231 (2019).

140. X. Dong, Y. Shi, W. Huang, P. Chen, and L.J. Li, Adv. Mater. 22, 1649 (2010).

141. R. Campos, J. Borme, J.R. Guerreiro, G. Machado, M.F. Cerqueira, D.Y. Petrovykh, and P. Alpuim, ACS Sensors 4, 286 (2019).

142. R. Stine, J.T. Robinson, P.E. Sheehan, and C.R. Tamanaha, $A d v$. Mater. 22, 5297 (2010).

143. B. Cai, S. Wang, L. Huang, Y. Ning, Z. Zhang, and G.J. Zhang, ACS Nano 8, 2632 (2014)

144. C. Zheng, L. Huang, H. Zhang, Z. Sun, Z. Zhang, G.J. Zhang, and A.C.S. Appl, Mater. Interfaces 7, 16953 (2015).

145. B. Cai, L. Huang, H. Zhang, Z. Sun, Z. Zhang, and G.J. Zhang, Biosens. Bioelectron. 74, 329 (2015).

146. D.W. Lee, J. Lee, I.Y. Sohn, B.Y. Kim, Y.M. Son, H. Bark, J. Jung, M. Choi, T.H. Kim, C. Lee, and N.E. Lee, Nano Res. 8, 2340 (2015).

147. H.Y. Park, S.R. Dugasani, D.H. Kang, G. Yoo, J. Kim, B. Gnapareddy, J. Jeon, M. Kim, Y.J. Song, S. Lee, J. Heo, Y.J. Jeon, S.H. Park, and J.H. Park, Sci. Rep. 6, 1 (2016).

148. L. Yan, H. Shi, X. Sui, Z. Deng, and L. Gao, RSC Adv. 7, 23573 (2017).

149. S.M. Majd, A. Salimi, and F. Ghasemi, Biosens. Bioelectron. 105, 6 (2018).

150. K.M. Yasuhide Ohno, ${ }^{*}$ K. Maehashi, Y. Yamashiro, Nano Lett. 9, 3318 (2009).
151. G. Seo, G. Lee, M. J. Kim, S. H. Baek, M. Choi, K. B. Ku, C. S. Lee, S. Jun, D. Park, H. G. Kim, S. J. Kim, J. O. Lee, B. T. Kim, E. C. Park, and S. Il Kim, ACS Nano 14, 5135 (2020).

152. S. Myung, A. Solanki, C. Kim, J. Park, K.S. Kim, and K.B. Lee, Adv. Mater. 23, 2221 (2011).

153. D.J. Kim, I.Y. Sohn, J.H. Jung, O.J. Yoon, N.E. Lee, and J.S. Park, Biosens. Bioelectron. 41, 621 (2013).

154. L. Wang, Y. Wang, J.I. Wong, T. Palacios, J. Kong, and H.Y. Yang, Small 10, 1101 (2014).

155. J. Lee, P. Dak, Y. Lee, H. Park, W. Choi, M.A. Alam, and S. Kim, Sci. Rep. 4, 1 (2014).

156. A.P.S. Gaur, S. Sahoo, M. Ahmadi, S.P. Dash, M.J.F. Guinel, and R.S. Katiyar, Nano Lett. 14, 4314 (2014).

157. H. Nam, B.R. Oh, P. Chen, J.S. Yoon, S. Wi, M. Chen, K. Kurabayashi, and X. Liang, Appl. Phys. Lett. 107, 1 (2015).

158. Y.M. Lei, M.M. Xiao, Y.T. Li, L. Xu, H. Zhang, Z.Y. Zhang, and G.J. Zhang, Biosens. Bioelectron. 91, 1 (2017).

159. M. Hosseini and S. Babaee Touski, J. Electron. Mater. 49, 4085 (2020).

Publisher's Note Springer Nature remains neutral with regard to jurisdictional claims in published maps and institutional affiliations. 